\title{
Numerical Study on Acoustic Resonance Excitation in Closed Side Branch Pipeline Conveying Natural Gas
}

\author{
Liuyi Jiang $\mathbb{D}^{1}{ }^{1}$ Hong Zhang, ${ }^{2}$ Qingquan Duan, ${ }^{1}$ and Yulong Zhang $\mathbb{D}{ }^{1,3}$ \\ ${ }^{1}$ College of Safety and Ocean Engineering, China University of Petroleum, Beijing 102249, China \\ ${ }^{2}$ National Engineering Laboratory for Pipeline Safety, MOE Key Laboratory of Petroleum Engineering, \\ Beijing Key Laboratory of Urban Oil and Gas Distribution Technology, \\ China University of Petroleum (Beijing), Beijing 102249, China \\ ${ }^{3}$ Australian Maritime College, Newnham, Tasmania 7248, Australia
}

Correspondence should be addressed to Liuyi Jiang; jiangliuyi409@163.com

Received 23 April 2020; Revised 20 September 2020; Accepted 22 September 2020; Published 23 October 2020

Academic Editor: Sinniah Ilanko

Copyright (c) 2020 Liuyi Jiang et al. This is an open access article distributed under the Creative Commons Attribution License, which permits unrestricted use, distribution, and reproduction in any medium, provided the original work is properly cited.

Flow-induced acoustic resonance in the closed side branch of a natural gas pipeline can cause intensive vibration which threatens the safe operation of the pipeline. Accurately modeling this excitation process is necessary for a workable understanding of the genetic mechanism to resolve this problem. A realizable $k-\varepsilon$ Delayed Detached Eddy Simulation (DDES) model was conducted in this study to numerically simulate the acoustic resonance problem. The model is shown to accurately capture the acoustic resonance phenomenon and self-excited vibration characteristics with low calculation cost. The pressure pulsation component of the acoustic resonance frequency is gradually amplified and transformed into a narrowband dominant frequency in the process of acoustic resonance excitation, forming a so-called "frequency lock-in phenomenon." The gas is pressed into and out of the branch in sinusoidal mode during excitation. The first-order frequency, single vortex moves at the branch inlet following the same pattern. A quarter wavelength steady standing wave forms in the branch. The mechanism and characteristics presented in this paper may provide guidelines for developing new excitation suppression methods.

\section{Introduction}

Natural gas is a clean, efficient, and high-quality energy resource which is a preferred fuel by many countries across the globe $[1,2]$. The mileage of natural gas pipeline construction projects has rapidly increased alongside the demand for fuel in recent decades. For example, the length of natural gas pipeline inservice has reached $81,000 \mathrm{~km}$ in China; $3,233 \mathrm{~km}$ of this length was newly built in 2019. The closed side branch of a natural gas pipeline is a commonly used unit of gas transmission. Flowinduced acoustic resonance occurs as gas flows through the mouth of a closed branch at a certain velocity, causing intensive and continuous gas pressure pulsations that may result severe pipeline vibration and even fatigue damage [3-6]. These pulsations are also noisy and intense enough to endanger the physical and mental health of workers. A serious acoustic resonance event occurred in the WKC2 gas transmission station of China in 2012 which fractured a tee connector (Figure 1).
There is substantial practical importance in accurately, comprehensively understanding the mechanism of acoustic resonance and the most effective approaches to suppressing it [7-12]. The essential mechanism of acoustic resonance in the closed side branch is the coupling effect of the flow field and sound field. The shear layer detaches from the upstream pipe wall, exciting a sound vortex that travels downstream and is coupled with the gas in the side branch; this causes acoustic vortex convection along the branch. The sound vortex is gradually excited as it detaches. The convection of the vortex (vortices) generates and absorbs sound energy to maintain continuous pressure pulsation [13].

The key to effectively understanding the shear layer detachment process lies in accurately modeling the sound source excitation and hydrodynamic modal in the branch mouth. Deriving a solvable differential equation of sound source excitation analytically is almost impossible, as the turbulence flow in the main pipe is excessively complex. The vortex acoustic 


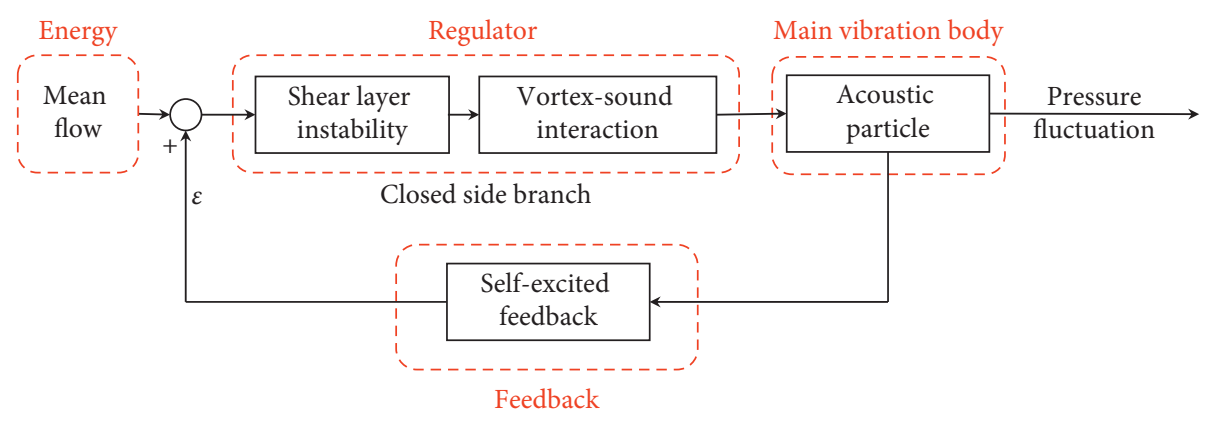

Figure 1: Schematic diagram of feedback mechanism.

theory developed by Powell [14] and Howe [15] has been widely used in the acoustic field, including acoustic resonance problems, but it is still difficult to solve vortex acoustic equations. Michalke [16] used linear stability theory to model a shear layer flow. Karlsson and Åbom [17] developed expressions for the side branch acoustic impedance under various velocities, and investigated the interaction between acoustic impedance and sound source excitation. Considering the nonlinearity of this problem, Graf and Ziada [18] proposed a semiempirical model for predicting the amplitude and phase of pressure fluctuations in tandem side branches and coaxial branches. Their model includes sound source excitation as the nonlinear pressure difference between the upper side and lower side of the detached shear layer in the branch mouth and can predict the acoustic amplitude and frequency of the pressure in the two branches.

Visual flow experiments and computational fluid dynamics (CFD) are the most common approaches to hydrodynamic modal analysis. In visual experiments, the morphology of the acoustic vortex and flow patterns of the fluid during the acoustic resonance can be clearly observed via high-speed camera. Ziada [19], for example, found that the mechanism of acoustic resonance is different from that of nonresonant oscillation of impact shear flow; visual analysis revealed that pressure fluctuation in closed side branches was caused by the coupling of the unstable detachment of shear layer from the upstream wall and standing acoustic waves in the side branch. During the unstable detachment of the shear layer, an acoustic vortex is excited as it is trapped in the mouth of the side branch. Li et al. [20-22] studied the flow patterns of acoustic resonance in tandem side branches and coaxial side branches using a high timeresolved particle image velocimetry (PIV) technique. They captured the velocity distribution in the flow field and constructed a phase delay diagram. Salt et al. [23, 24] also used PIV to observe the acoustic energy transfer pattern during an acoustic resonance cycle as per the sound source generation and absorption with shear layer oscillation. Xiao et al. [25] similarly obtained a multimodal image of shear layer detachment under the acoustic resonance of a square single closed side branch.

Flow patterns in closed side branches can be analyzed effectively via CFD methods. For example, Tamura et al. [26] modeled the acoustic resonance in three side branches based on a three-dimensional (3D) model using the finite difference lattice Boltzmann method. Kriesels et al. [5] simulated the gas flow and calculated the sound power generated by a vortex using the vortex-blob method based on a $2 \mathrm{D}$ potential flow model. Radavich et al. [27] simulated the 2D flow of a single closed side branch by solving Navier-Stokes equations of unsteady and compressible turbulence. Dequand et al. [28] simulated a 2D compressible coaxial closed side branch flow via Euler equation. Numerical simulations accurately predicted the range of frequency lock-in velocity and flow patterns.

Previous research has mainly centered on the formation conditions, pressure fluctuation amplitudes, and frequencies of acoustic resonance. Few scholars have modeled the acoustic resonance excitation process. Radavich et al. [27] briefly discussed the coupling of sound waves and flow in the excitation process and simulated the variations in pressure pulsation during excitation, but there has been no extensive research on the mechanism and excitation process of acoustic resonance. Such knowledge has great significance in regards to establishing an accurate, descriptive model.

This paper presents a numerical simulation of the excitation process of acoustic resonance in a closed side branch of natural gas pipeline using the commercial CFD code FLUENT. Due to safety concerns, researchers often use air instead of natural gas as a fluid medium in physical tests. Our findings show that the ingredients of the fluid medium have a pronounced impact on acoustic resonance characteristics.

Figure 1 shows a functional block diagram of the feedback mechanism of the system under analysis here. The red dotted frame marks the self-excitation system given by Ding [29], and the black solid frame is the feedback mechanism of acoustic resonance in the closed side branch. The mean flow kinetic energy in the main pipeline provides constant energy for the system. The closed side branch structure is a regulator. The acoustic particles in the pipe are the main vibration body. The pressure fluctuation (acoustic standing wave) in the closed side branch is fed back to the regulator to control the continuity of the fluctuation. The flow-induced acoustic resonance has self-excited vibration characteristics, so a self-excited vibration model was used in this study.

\section{Mathematical Models}

2.1. Geometry Model Simplification. Figure 2(a) shows a typical closed side branch of a natural gas pipeline in which acoustic resonance occurs. Considering its actual dimensions, which are usually larger than $0.5 \mathrm{~m}$ in diameter and 


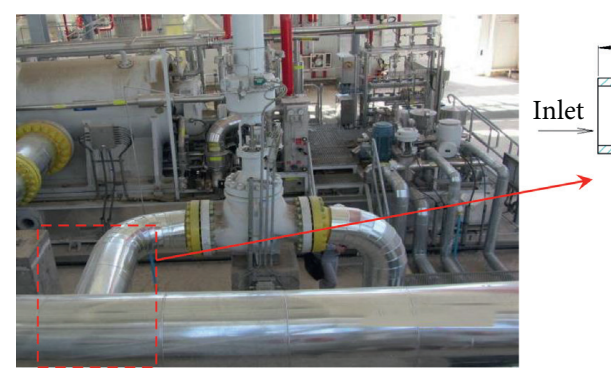

(a)

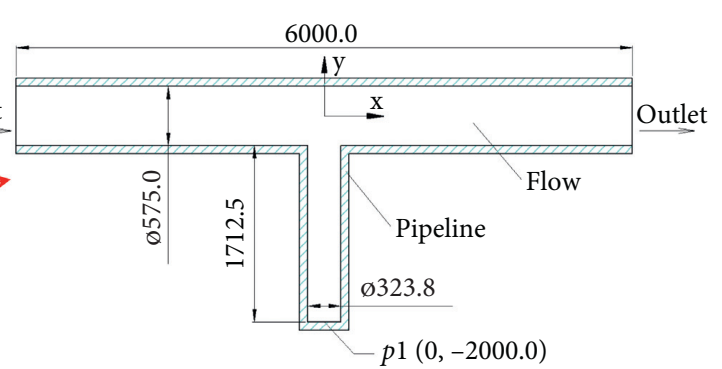

(b)

FIGURE 2: Schematic diagram showing the geometry of the closed side branch of a natural gas pipeline: (a) physical structure of the branch pipe in situ; (b) simplified geometry of the branch pipe (unit: $\mathrm{mm}$ ).

$1.5 \mathrm{~m}$ in length, the elbow at the end of the side branch can be neglected. The simplified geometry of closed side branch is shown in Figure 2(b). The model consists of two parts: the main pipe and the closed side branch. The length and inner diameter of the main pipe are $6000.0 \mathrm{~mm}$ and $575.0 \mathrm{~mm}$, respectively. The branch pipe is located at the center of the main pipe and has a length and inner diameter of $1712.5 \mathrm{~mm}$ and $323.8 \mathrm{~mm}$, respectively. The flow medium is methane (the main component of natural gas), which is input steadily from the inlet side and flows continuously to the outlet side. The coordinate system is shown in Figure 2(b), where the $x$ axis falls along the center line of the main pipe and the $y$-axis along the center line of the branch pipe. The pressuremonitoring point $p 1(0,-2000,0)$ is located at the bottom of the center of the side branch.

2.2. CFD Turbulence Model Configuration. Reynolds Average Navier-Stokes (RANS), Detached Eddy Simulation (DES), and Large Eddy Simulation (LES) $[30,31]$ are the most commonly used CFD models. Among them, RANS is most often applied due to its minimal computational cost. However, it is often unable to accurately predict pressure fluctuations in transient flow fields. We found that the pressure fluctuation amplitude of acoustic resonance in a closed side branch, as calculated by RANS, gradually decreases over time and ultimately attenuates to 0 . We considered a hybrid model of the subregional computation of RANS and LES, in this study, so DES was a workable solution. DES solves near-wall flow field by RANS and other flow fields by LES, thus avoiding the need for an overly fine grid near the LES wall and striking a balance between numerical calculation cost and accuracy [32]. In near-wall flow fields, a realizable $k-\varepsilon$ model can accurately simulate boundary-free shear flows [33]. A realizable $k-\varepsilon$ DDES model [34-36] was used in this study to investigate acoustic resonance in the closed side branch.

\section{Governing Equations for the Acoustic Resonance Process}

3.1. Conservation Equations. The mass, momentum, and energy conservation equations as well as ideal gas law that the acoustic resonance process complies with can be written as follows:

$$
\begin{aligned}
\frac{\partial \rho}{\partial t}+\frac{\partial}{\partial x_{j}}\left(\rho u_{i}\right) & =0 \\
\frac{\partial}{\partial t}\left(\rho u_{i}\right)+\frac{\partial}{\partial x_{j}}\left(\rho u_{i} u_{j}\right) & =-\frac{\partial p}{\partial x_{i}}+\frac{\partial \tau_{i j}}{\partial x_{j}} \\
\frac{\partial}{\partial t}(\rho E)+\frac{\partial}{\partial x_{i}}\left(u_{i}(\rho E+p)\right) & =\frac{\partial}{\partial x_{i}}\left(k_{\mathrm{eff}} \frac{\partial T}{\partial x_{i}}-h J+u_{j}\left(\tau_{i j}\right)_{\mathrm{eff}}\right), \\
p & =\rho T \frac{R}{M},
\end{aligned}
$$

where $\rho$ is the gas density, $t$ is the time, $p$ is the pressure, $\tau$ is the viscous stress tensor, $E$ is the sum of internal energy and kinetic energy, $T$ is the temperature, $h$ is the gas enthalpy, $J$ is the diffusion energy, $k_{\text {eff }}$ is the effective thermal conductivity, $\tau_{\text {eff }}$ is the effective viscous dissipation coefficient, $R$ is the universal gas constant, and $M$ is the molecular weight.

3.2. Transport Equations. The flows discussed here are characterized by high Reynolds number turbulence, as their Reynolds numbers range from $2.03 \mathrm{E}+07$ to $1.42 \mathrm{E}+08$. Additional transport equations are needed to construct the turbulence closure models. The DDES model adopts the following dual realizable $k$ - $\varepsilon$ equations [34]:

$$
\begin{aligned}
\frac{\partial(\rho k)}{\partial t}+\frac{\partial\left(\rho k u_{i}\right)}{\partial x_{i}}= & \frac{\partial}{\partial x_{j}}\left[\left(\mu+\frac{\mu_{t}}{\sigma_{k}}\right) \frac{\partial k}{\partial x_{j}}\right]+G_{k}-\rho \varepsilon-Y_{M}, \\
\frac{\partial(\rho \varepsilon)}{\partial t}+\frac{\partial\left(\rho \varepsilon u_{i}\right)}{\partial x_{i}}= & \frac{\partial}{\partial x_{j}}\left[\left(\mu+\frac{\mu_{t}}{\sigma_{\varepsilon}}\right) \frac{\partial \varepsilon}{\partial x_{j}}\right]+\rho C_{1 \varepsilon} S \varepsilon \\
& -C_{2 \varepsilon} \rho \frac{\varepsilon^{2}}{k+\sqrt{v \varepsilon}}+C_{1 \varepsilon} \frac{\varepsilon}{k} C_{3 \varepsilon} G_{b},
\end{aligned}
$$

where $Y_{M}=\rho k^{3 / 2} / l_{\text {des }}, l_{\text {des }}=l_{r k e}-f_{d} \max \left(0, l_{r k e}-C_{d e s} \Delta_{\max }\right)$ $l_{r k e}=k^{3 / 2} / \varepsilon, \quad f_{d}=1-\tanh \left(20 r_{d}\right)^{3}, \quad r_{d}=\left(v_{t}+\right.$ $v) /\left(\sqrt{U_{i, j} U_{i, j}} k^{2} d\right), \quad \mu_{t}=\rho C_{\mu} k^{2} / \varepsilon, \quad C_{\mu}=1 /\left(A_{0}+A_{S} k v^{2} / \varepsilon\right)$, $A_{0}=4.04, A_{S}=\sqrt{6} \cos \phi, \phi=1 / 3 \cos ^{-1}(\sqrt{6} W), W=\left(S_{i j} S_{j k}\right.$ $\left.S_{k i} / S\right), \quad S=\sqrt{2 S_{i j} S_{i j}}, \quad S_{i j}=1 / 2\left(\left(\partial u_{j} / \partial x_{i}\right)+\left(\partial u_{i} / \partial x_{j}\right)\right)$, 
$C_{1 \varepsilon}=\max 0.43,(\eta /(\eta+5)), \quad C_{2 \varepsilon}=1.9, \quad C_{3 \varepsilon}=\tanh |v / u|$, $\eta=S k / \varepsilon, G_{k}$ denotes the turbulent kinetic energy due to the mean velocity, $\operatorname{Pr}_{t}=0.85$ in the realizable $k-\varepsilon$ model, $C_{d e s}=0.61$, and $\Delta_{\max }$ is the grid spacing, which in the case of a rectilinear hexahedral cell is the maximum edge length. In the region far away from the wall, $r_{d}$ is far less than 1 and $f_{\mathrm{d}}$ is equal to 1 , then the governing equation changes to LES; in the region near the wall, $f_{\mathrm{d}}$ is equal to 0 , and then the governing equation is in the RANS form of the realizable $k$-e model. In short, $Y_{M}$ thus controls the transformation of RANS to LES.

3.3. Grid Partition. A hexahedral structured grid was selected to improve the accuracy and convergence of the computation. All block grids in the pipeline were made O-shaped to improve the quality of the grid, as shown in Figure 3. The grid was refined in the side branch mouth and parts adjacent to the main pipe as the acoustic vortices are mainly located in these areas when acoustic resonance occurs. The thickness of the first layer near the wall was set to $0.04 \mathrm{~mm}$, and the internal area grid increased inward at a growth rate of 1.2 .

3.4. Boundary Conditions and Solver. The inlet boundary condition was considered a velocity inlet. The inlet velocities of all cases were configured with a mean flow and without fluctuation (Table 1) and represented by Strouhal number $S_{t}$. The Strouhal number is a dimensionless number that characterizes the unsteady similarity of fluid flow and is calculated as $S_{t}=f d / v$, where $d$ is the mouth diameter of the side branch, $v$ is the mean flow velocity of the main pipe, and $f$ is the first-order acoustic resonance frequency calculated as follows [37]:

$$
f_{n}=\frac{(2 n-1) c}{4(L+L e)}
$$

$n=1,2,3, \ldots$, is the order of the frequency, $c$ is the flow field sound velocity, $L$ is the branch length, and $L e$ is correction factor, 0.4-0.425 times the diameter of the branch.

The outlet boundary condition was considered a pressure outlet with a pressure of $0 \mathrm{~Pa}$ and operating pressure of $6 \mathrm{MPa}$. The methane gas $\left(\mathrm{CH}_{4}\right.$, the main component of natural gas) in the pipe was assumed to be compressible ideal gas. The wall temperature was set to 293.15 K. The walls were designed as rigid and nonpermeable, so heat does not transfer through them. It should be noted that although there was no heat exchange between the fluid and the environment through the wall in the simulation, energy conversion did exist in the flow (e.g., the conversion of pressure potential energy to heat energy, molecular friction leading to heat generation), so equation (3) is a necessary component of the CFD model.

The PISO algorithm coupled with neighbour and skewness corrections was adopted as the conservation equation solver. The conservation equations were spatially discretized by the finite volume method with the discretization schemes shown in Table 2.
The parameters of the mean flow velocity, pressure, and flow time were normalized as the dimensionless parameters Strouhal number $S t$, normalized pressure $p^{*}$, and normalized time $t^{*}$, respectively. These parameters are calculated as follows:

$$
\begin{aligned}
S t & =\frac{f d}{v}, \\
p^{*} & =\frac{p}{(1 / 2) \rho v^{2}}, \\
t^{*} & =\frac{v t}{l},
\end{aligned}
$$

where $f$ is the first-order frequency of acoustic resonance, $d$ is the diameter of the side branch, $v$ is the mean flow velocity, $p$ is the pressure amplitude, $\rho$ is the gas density, $t$ is the flow time, and $l$ is the distance from the inlet to the branch.

\section{Validation}

4.1. Grid Independent Solution Validation. It is necessary to verify the grid independence to prevent grid quality from affecting the accuracy of the numerical computation. For grid independence verification, the number of adjacent grids is usually set to be approximately twice as large as that of adjacent grids $[38,39]$. Four sets of grids were prepared to compute with the boundary condition with a $40 \mathrm{~m} / \mathrm{s}$ inlet velocity. The cell number of grids was about 159151, 354435, 790423, and 1494845, respectively. All the cases were simulated to $2 \mathrm{~s}$, at which point the quasisteady state of acoustic resonance was reached. The pressure fluctuation at the monitoring point $p 1$ of the four sets of grids is shown in Figure 4(a). The dominant frequency of all pressure fluctuations was equal to $50.6 \mathrm{~Hz}$ by FFT analysis, which shows that the four sets of grids did not affect the pressure fluctuation frequency in this case.

As shown in Figure 4(a), the pressure fluctuation waveforms of 159151 grids have larger errors than those of other grids while the pressure fluctuation results of 354435, 790423, and 1494845 grids are basically the same, with nearly coincident curves. The amplitude and phase of the dominant frequency pressure fluctuation of four sets of grids were obtained by FFT as shown in Figure 4(b), where though the pressure fluctuations of the four sets of grids are similar, the phase differences among them are stark. There are small phase changes in the 790423 and 1494845 grids, so 790 and 423 grids can be considered grid independent solutions.

4.2. Frequency Validation. According to equation (7), $f_{1}=64.7 \mathrm{~Hz}$ and $f_{3}=194.1 \mathrm{~Hz}$ based on the simulated flow conditions (the natural gas adiabatic index $K=1.479$, and the gas constant $R=518.2 \mathrm{~J} /(\mathrm{kg} \cdot \mathrm{K})$. The sound velocity $c=471.4 \mathrm{~m} / \mathrm{s}$ as per $c=\sqrt{K R T}$. The simulation results show a first-order frequency $f_{1 \mathrm{~s}}=50.8 \mathrm{~Hz}$ at the mean flow velocity of $v=20 \mathrm{~m} / \mathrm{s}$ and a first-order frequency $f_{2 \mathrm{~s}}=185.0 \mathrm{~Hz}$ at the mean flow velocity of $v=70 \mathrm{~m} / \mathrm{s}$. These theoretical calculation results are in good agreement with the simulation results. 


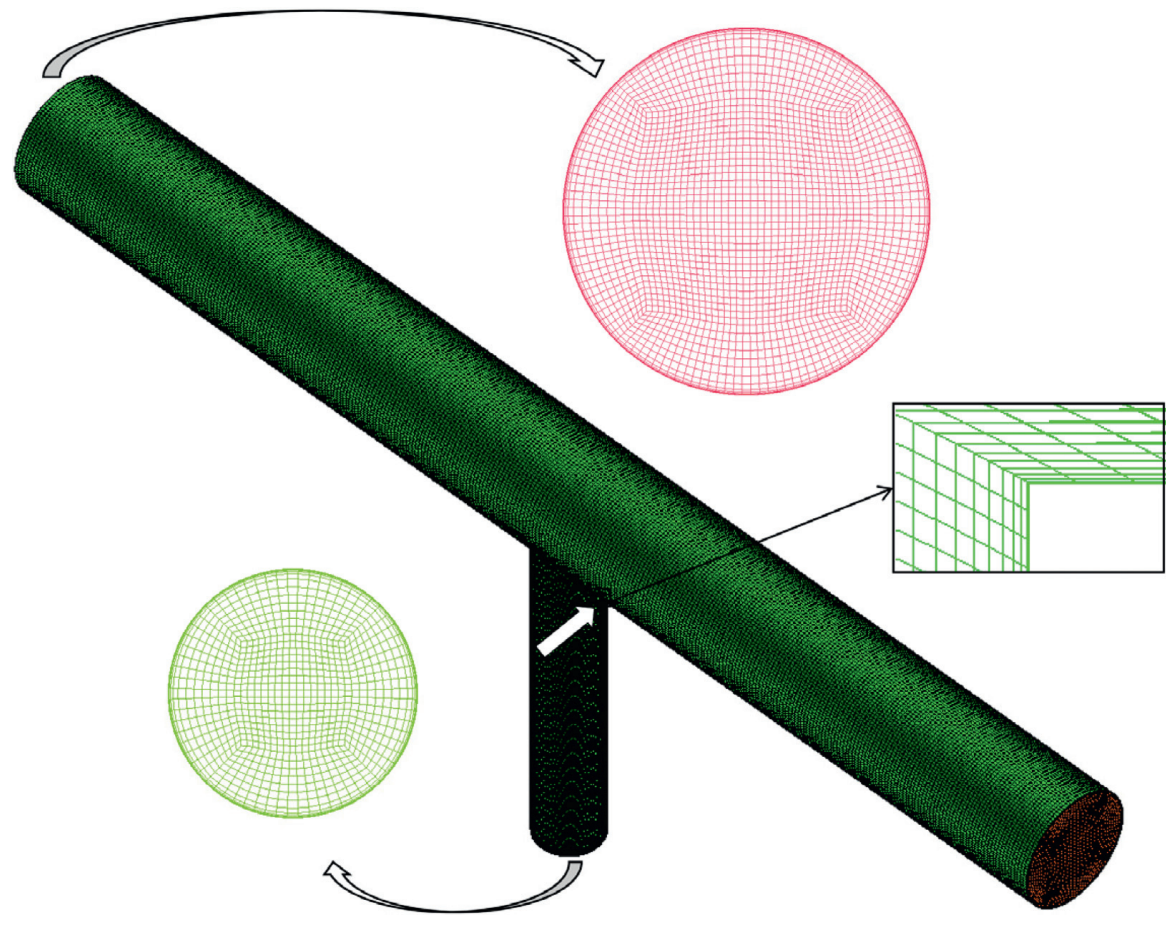

Figure 3: Grid area.

TABLE 1: Parameters of the cases.

\begin{tabular}{lccc}
\hline Case & Velocity $(\mathrm{m} / \mathrm{s})$ & $S t$ & $R e$ \\
\hline 1 & 10 & 2.00 & $2.03 E+07$ \\
2 & 15 & 1.34 & $3.04 E+07$ \\
3 & 20 & 1.00 & $4.05 E+07$ \\
4 & 30 & 0.67 & $6.08 E+07$ \\
5 & 40 & 0.50 & $8.11 E+07$ \\
6 & 50 & 0.40 & $1.01 E+08$ \\
7 & 60 & 0.33 & $1.22 E+08$ \\
8 & 70 & 0.29 & $1.42 E+08$ \\
\hline
\end{tabular}

TABLE 2: Spatial discretization schemes for the governing equations.

\begin{tabular}{lc}
\hline Governing equations & Schemes \\
\hline Gradient & Least squares cell-based \\
Pressure & Second order \\
Momentum & Bounded central difference \\
Turbulent kinetic energy & Second-order upwind \\
Turbulent dissipation rate & Second-order upwind \\
Energy & Second-order upwind \\
\hline
\end{tabular}

4.3. Experimental Validation. It is difficult to do experiments under pressure as high as $6 \mathrm{MPa}$. We compared our simulation results of $0.3 \mathrm{MPa}$ with the experimental results of Ziada Shine [11]. As shown in Figure 5, the CFD simulation pressure is generally higher than the experimental pressure, as the wall was rigid and impenetrable in the CFD simulation. The pipe absorbed vibration energy in the experiment, however, so the acoustic energy could propagate outward through the pipe wall. The simulation result is basically consistent with the experimental result at the Strouhal number $(S t)$ range of acoustic resonance, which indicates that our model can effectively simulate the acoustic resonance in the closed side branch.

\section{Results and Analysis}

5.1. Pressure Fluctuation and FFT Analysis. Figure 6 shows the time history of pressure fluctuation in monitoring point $p 1$ at different mean flow velocities. All of these cases were initialized from the inlet of the main pipe at the corresponding velocity and were calculated until a stable acoustic resonance formed. The acoustic resonance excitation process initially attenuated at various flow velocities, presenting cluttered signals. The difference was whether acoustic resonance finally formed. For $S t>1$, as shown in Figures 6(a) and 6(b)), acoustic resonance did not occur and the pressure fluctuation amplitude was low. For $S t<1$, as shown in Figures 6(c)-6(h)), acoustic resonance did occur and the amplitude of pressure fluctuation was higher. It appears to take some time for stable acoustic resonance to form, less time when the mean flow velocity is relatively large.

For main pipe mean flow velocity of $v>40 \mathrm{~m} / \mathrm{s}$, the pressure fluctuation shows beat vibration characteristics indicative of unstable self-excited vibration, though continuous fluctuations were maintained in this case.

According to the FFT analysis (Figure 7), all the cases contain a frequency component of approximately $50 \mathrm{~Hz}$. More low frequency, small amplitude components were observed when mean flow velocity $v=10 \mathrm{~m} / \mathrm{s}$ and $20 \mathrm{~m} / \mathrm{s}$ (Figures 5 (a) and 5(b)). Although there is a component of approximately $50 \mathrm{~Hz}(f=49.1 \mathrm{~Hz}$ and $48.7 \mathrm{~Hz}$ correspondingly), the amplitude is much smaller than that of other cases. In the case of the 


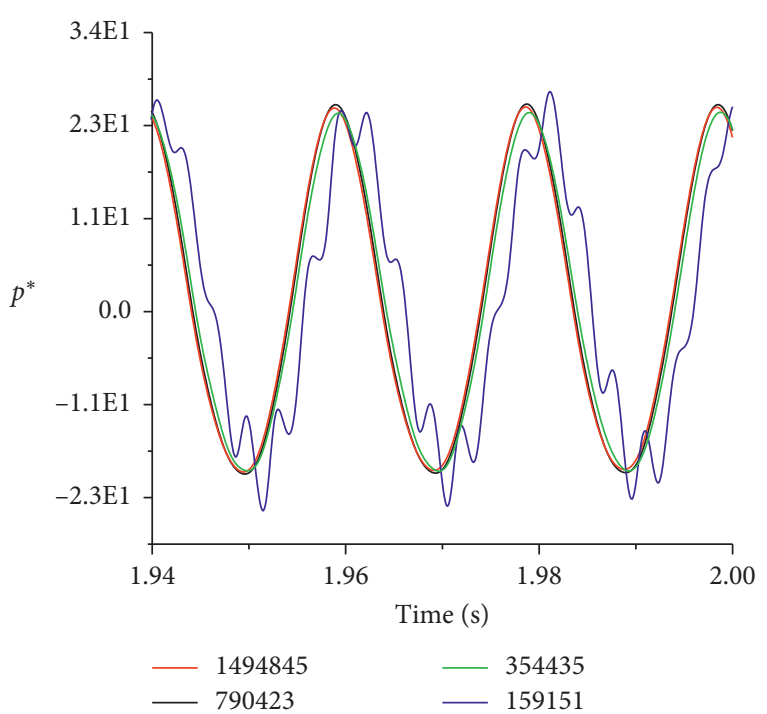

(a)

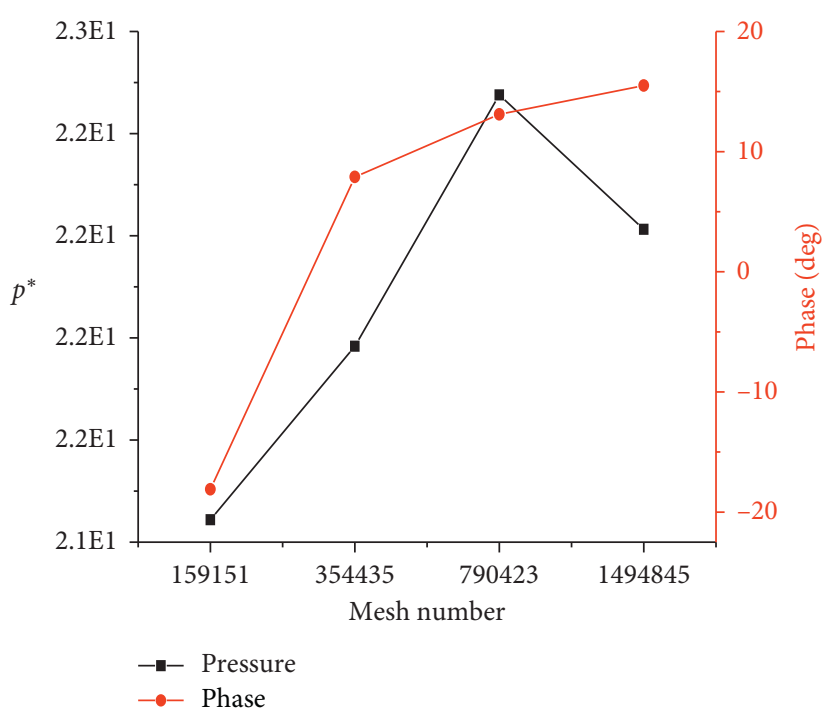

(b)

FIGURE 4: Verification for grid independent solutions.

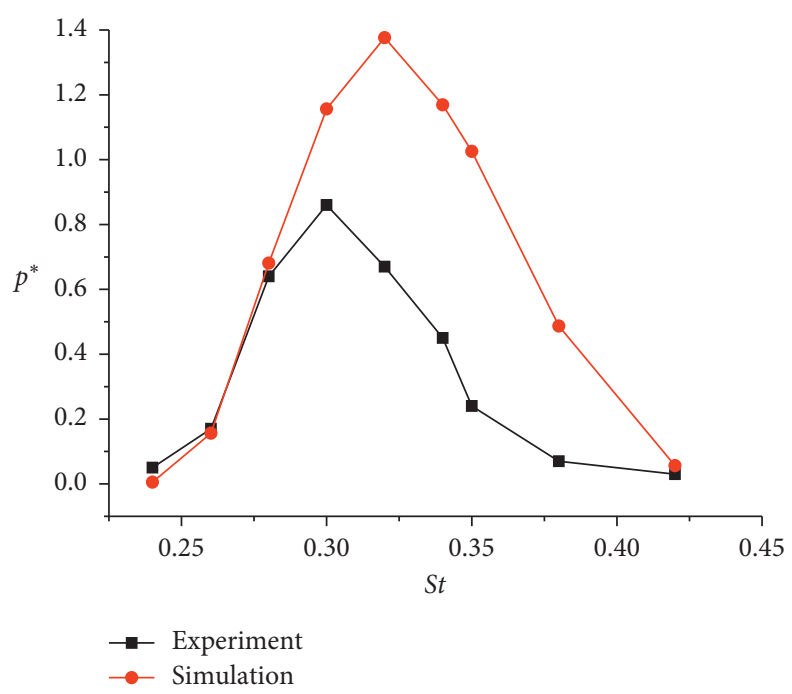

FIgUre 5: Comparison between Ziada and Shine [11] results and current simulation results.

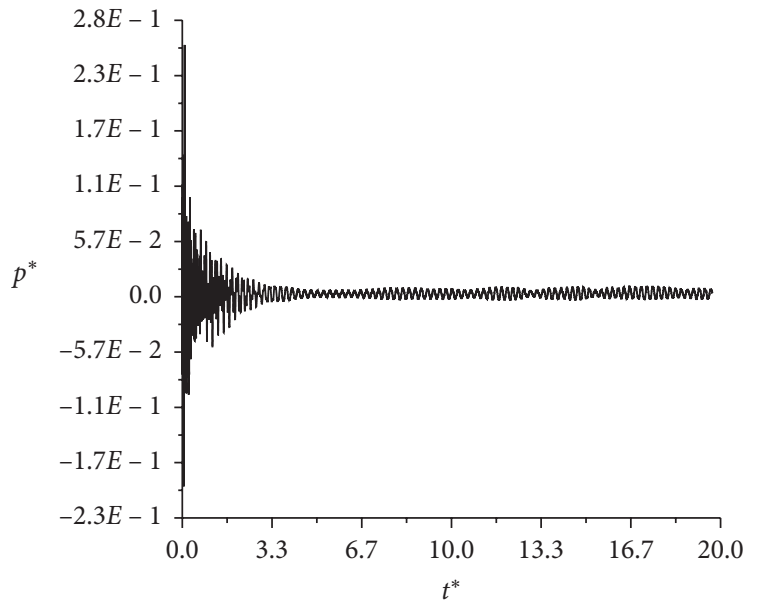

(a)

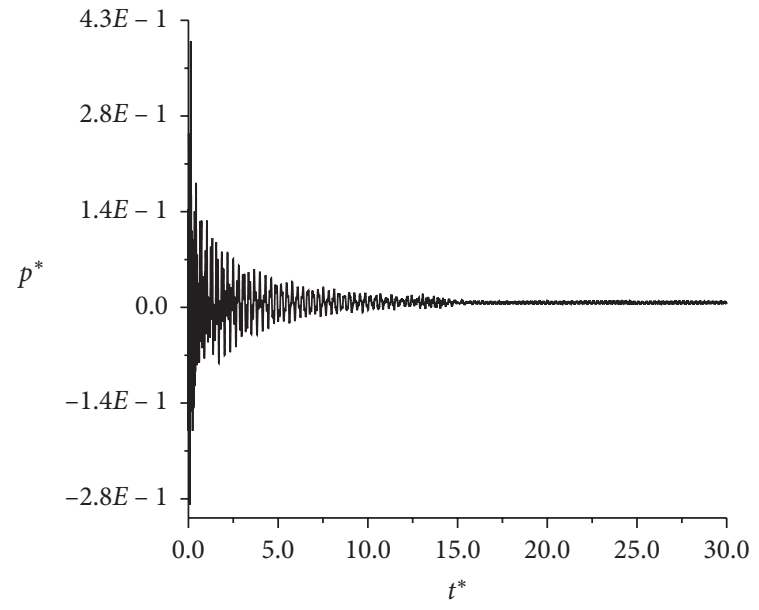

(b)

Figure 6: Continued. 


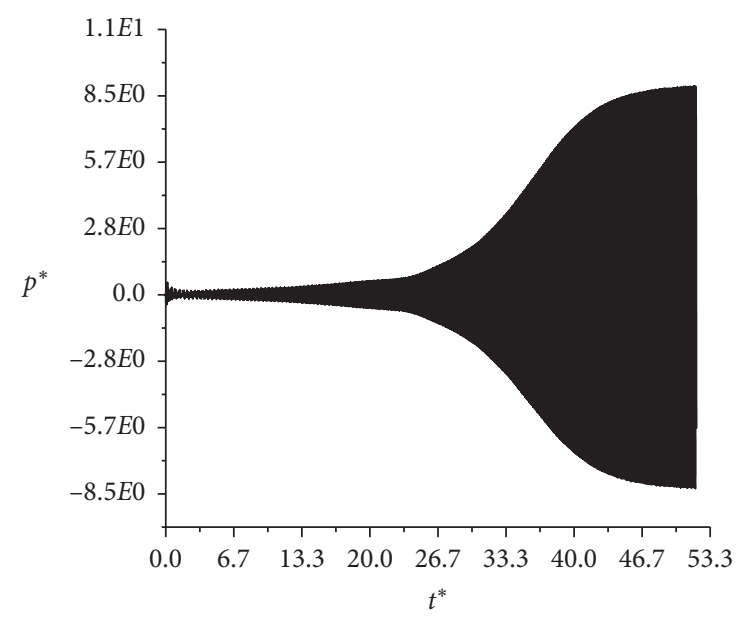

(c)

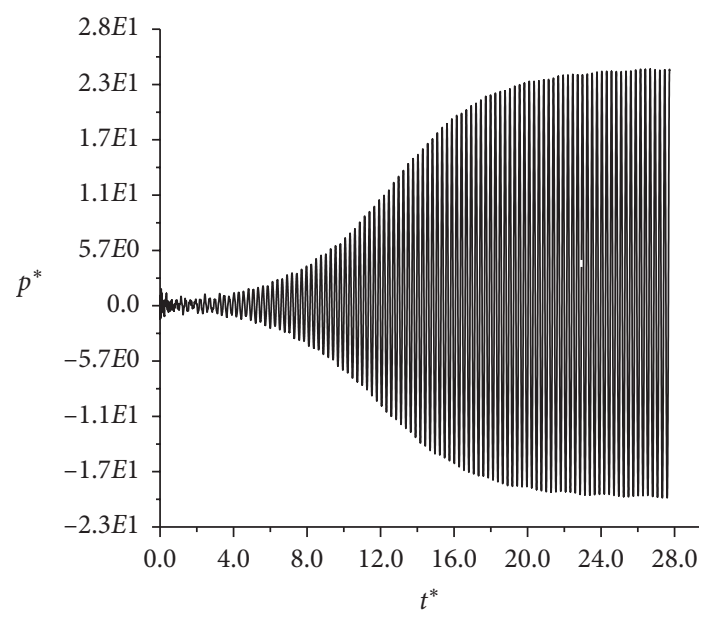

(e)

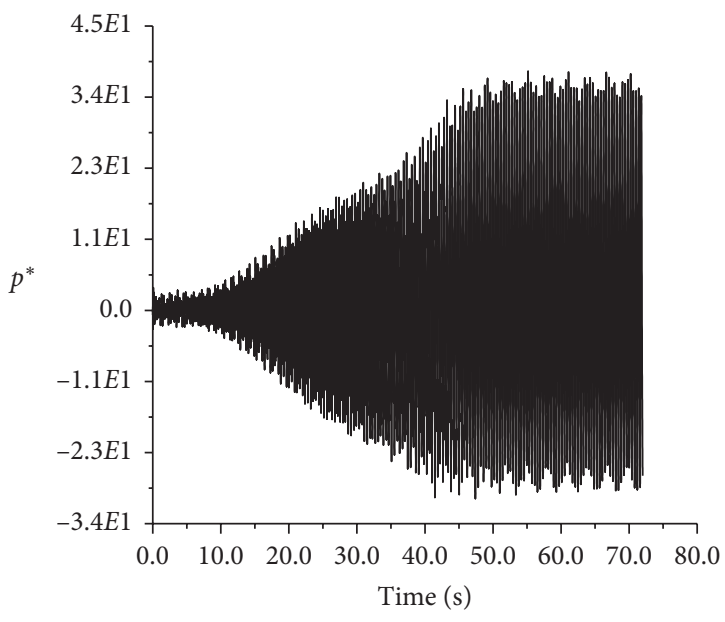

(g)

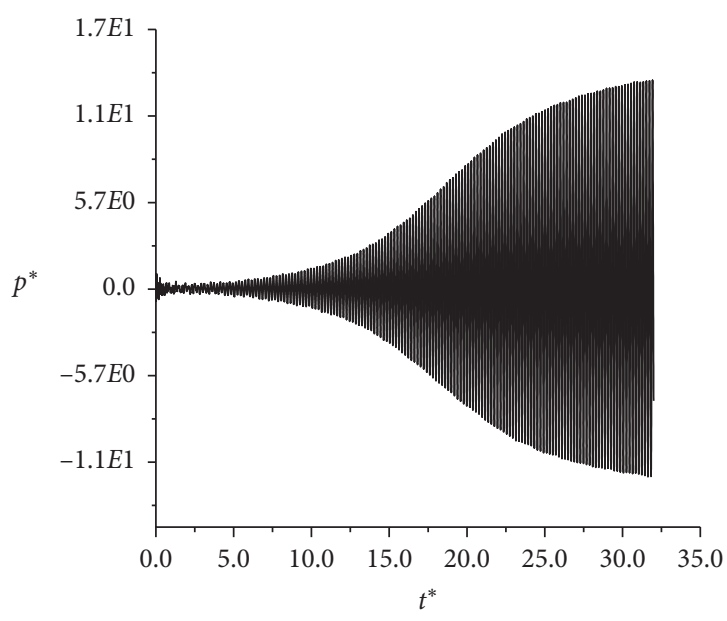

(d)

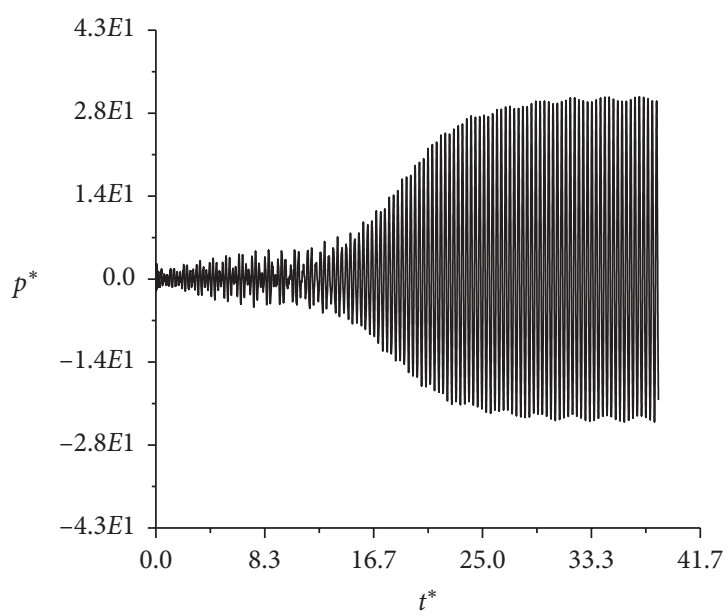

(f)

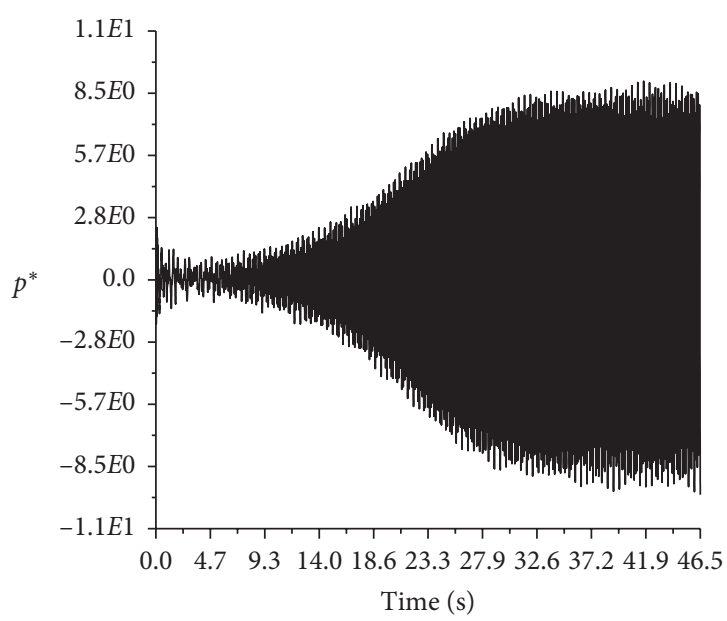

(h)

Figure 6: Pressure fluctuation: (a) $v=10 \mathrm{~m} / \mathrm{s}$, (b) $v=15 \mathrm{~m} / \mathrm{s}$, (c) $v=20 \mathrm{~m} / \mathrm{s}$, (d) $v=30 \mathrm{~m} / \mathrm{s}$, (e) $v=40 \mathrm{~m} / \mathrm{s},(\mathrm{f}) v=50 \mathrm{~m} / \mathrm{s}$, (g) $v=60 \mathrm{~m} / \mathrm{s}$, and (h) $v=70 \mathrm{~m} / \mathrm{s}$.

mean flow velocity $v=20 \mathrm{~m} / \mathrm{s}, 30 \mathrm{~m} / \mathrm{s}, 40 \mathrm{~m} / \mathrm{s}$, and $50 \mathrm{~m} / \mathrm{s}$ (Figures 5(c)-5(f)), the dominant frequency of pressure fluctuation was approximately $50.8 \mathrm{~Hz}$ of the first order of the acoustic resonance, with narrow frequency characteristics, while the other components had smaller amplitudes. This indicates a pure-tone acoustic resonance. In the case of the 


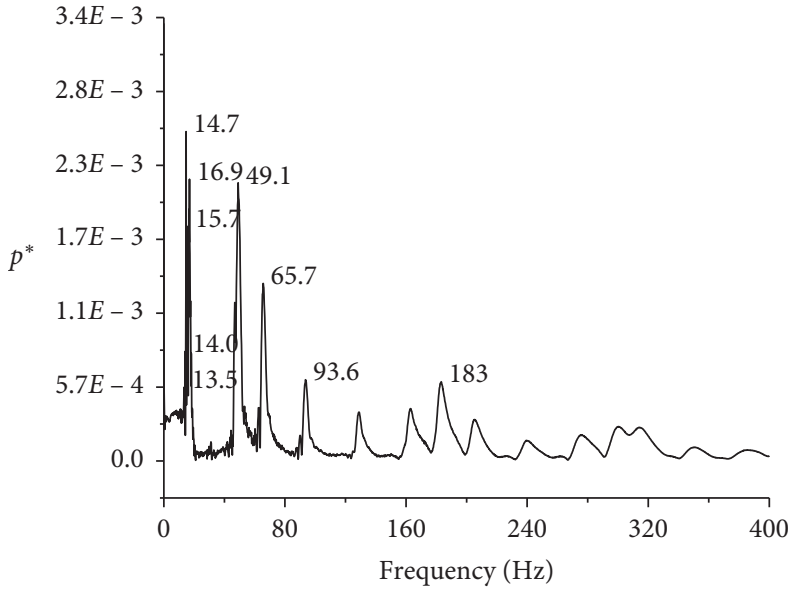

(a)

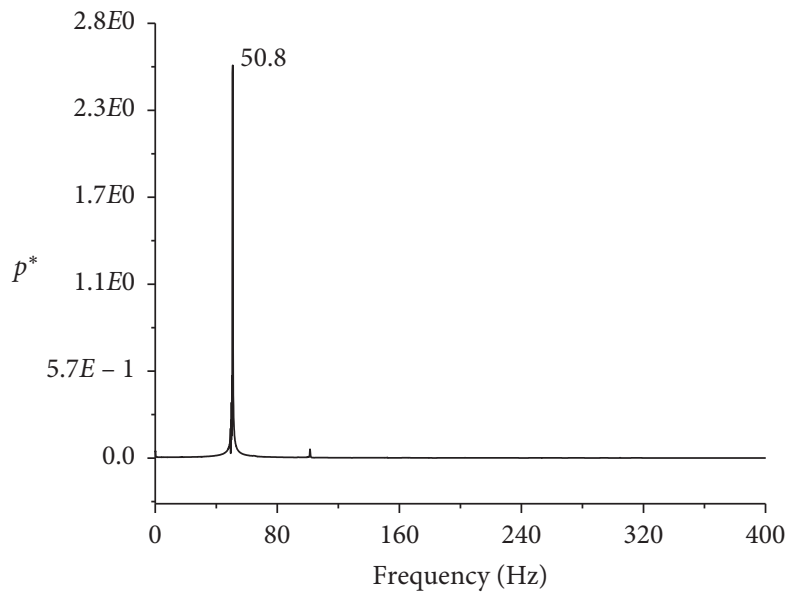

(c)

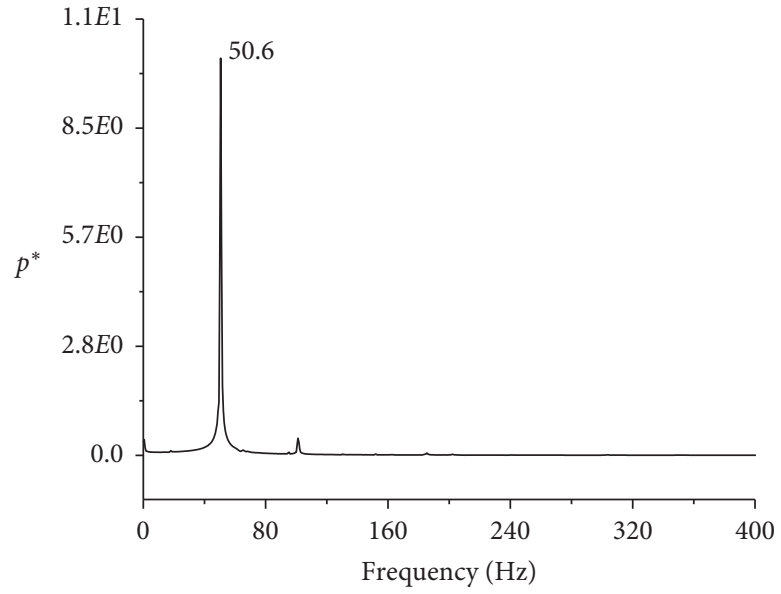

(e)

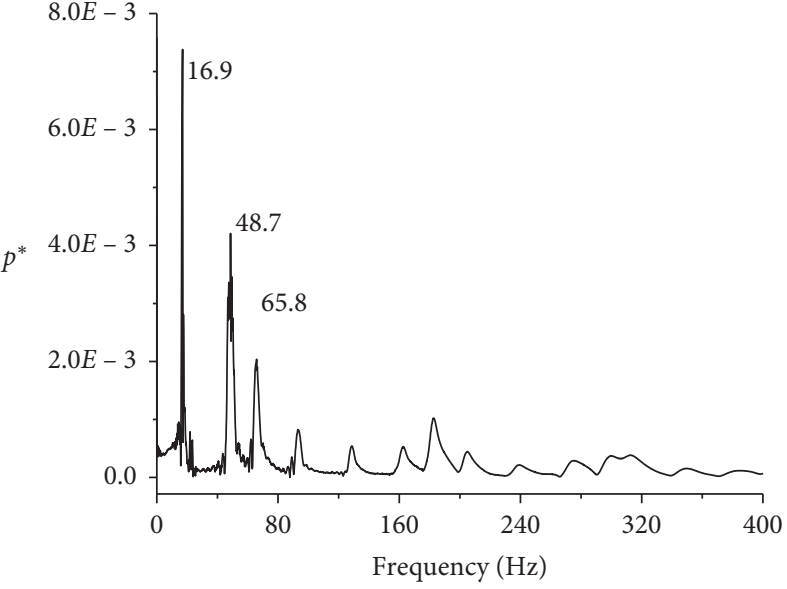

(b)

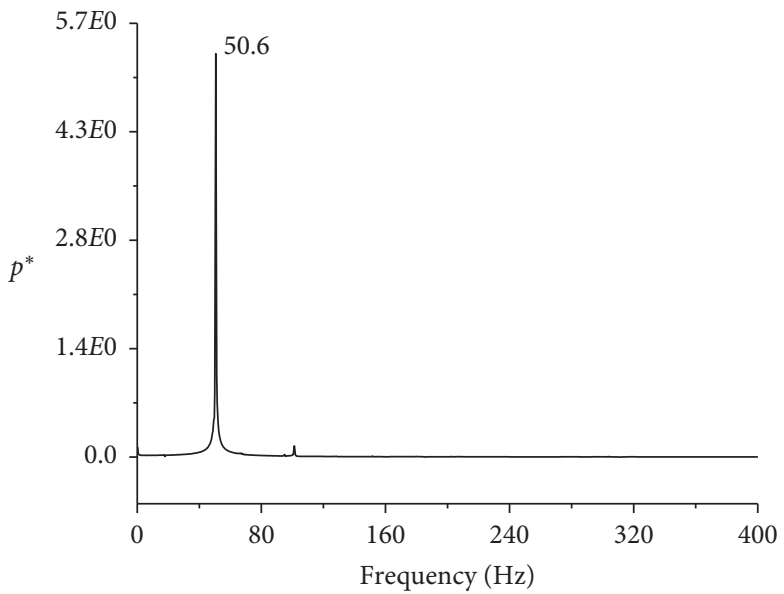

(d)

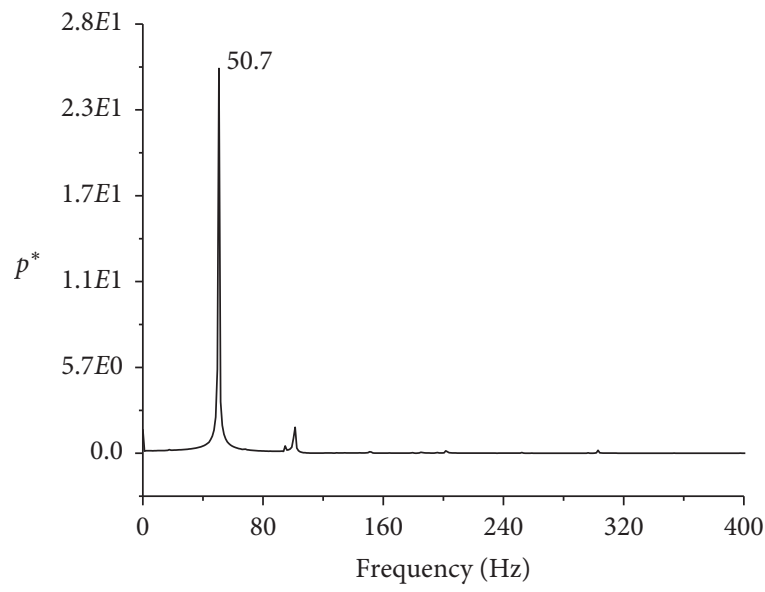

(f)

Figure 7: Continued. 


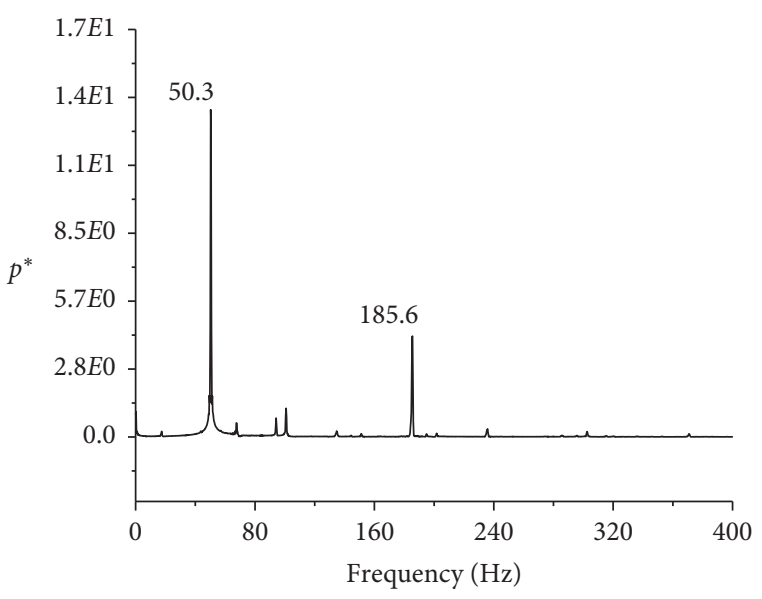

(g)

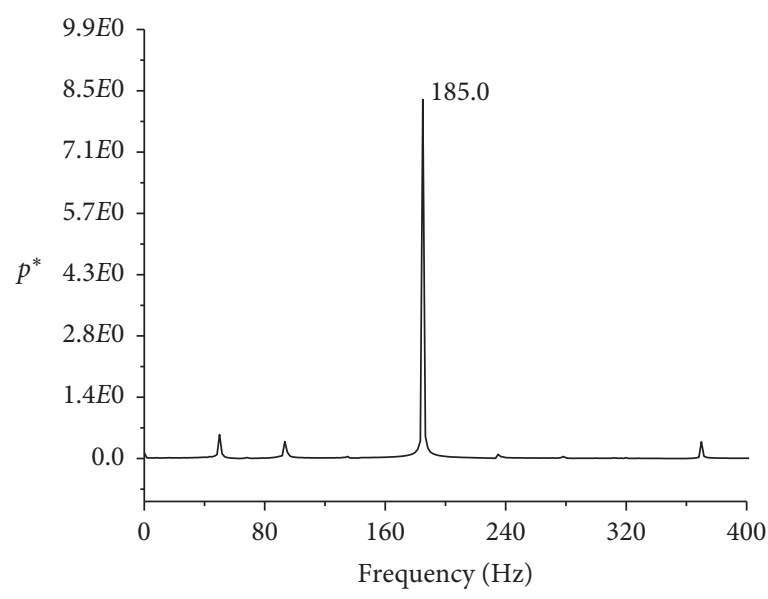

(h)

Figure 7: Pressure fluctuation FFT analysis: (a) $v=10 \mathrm{~m} / \mathrm{s}$, (b) $v=15 \mathrm{~m} / \mathrm{s}$, (c) $v=20 \mathrm{~m} / \mathrm{s}$, (d) $v=30 \mathrm{~m} / \mathrm{s}$, (e) $v=40 \mathrm{~m} / \mathrm{s}$, (f) $v=50 \mathrm{~m} / \mathrm{s}$, (g) $v=60 \mathrm{~m} / \mathrm{s}$, and (h) $v=70 \mathrm{~m} / \mathrm{s}$.

mean flow velocity $v=70 \mathrm{~m} / \mathrm{s}$ (Figure $7(\mathrm{~h})$ ), the dominant frequency of pressure fluctuation was $185.0 \mathrm{~Hz}$ of the second order, but the component amplitude of $50.7 \mathrm{~Hz}$ was not obvious; the acoustic resonance transformed into a pure tone of the second order. In the case of the mean flow velocity $v=60 \mathrm{~m} / \mathrm{s}$ (Figure $7(\mathrm{~g})$ ), unlike other cases where acoustic resonance occurred, the frequencies of pressure fluctuation contained two frequencies: a dominant frequency of $50.3 \mathrm{~Hz}$ and a secondary frequency of $185.6 \mathrm{~Hz}$. These results indicate that the mean flow velocity $v=60 \mathrm{~m} / \mathrm{s}$ fell in the transitional velocity range from the first-order acoustic resonance to the second. The order of the acoustic resonance frequency is defined by the parameter $n$ in equation (7).

\subsection{Frequency and Amplitude Characteristics of Excitation} Process. The dominant frequency and its pressure amplitude are presented in Figure 8 as function of $S t$ and velocity. The dominant frequencies in the cases of $0.3<S t<1$ showed a frequency lock-in phenomenon, which has been found in previous experimental studies. In the cases of $S t>1$, the dominant frequency and its amplitude were lower and the pressure fluctuation amplitude could be neglected. The pressure fluctuation amplitude reached its maximum when $S t=0.4$, which is consistent previous experimental results wherein the peak pressure fluctuation amplitude appears around St of 0.4 or 0.45 [37]. Previously published experiments have generally shown that acoustic resonance occurs in the range of $0.2<S t<0.6$ when the fluid medium is air. In the present study, acoustic resonance still occurred when $S t$ reaches 1.0 for the fluid medium of methane. This indicates that the fluid medium affects the value of $S t$ at which acoustic resonance occurs. The fluid medium of methane acoustic resonance occurs at a higher $S t$ (lower mean flow velocity) than air, so the natural gas pipeline is a riskier environment in terms of acoustic resonance in closed side branches.

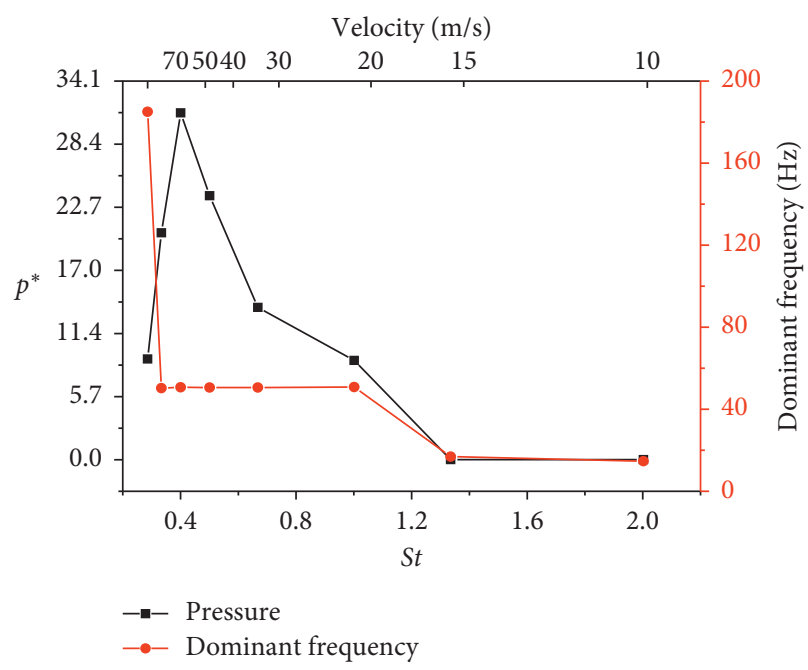

FIGURE 8: Dominant frequency (right) and pressure amplitude (left) as function of $S t$ (bottom) and velocity (top).

Figures 9(a)-9(e)) show the spectra corresponding to the time intervals $\Delta t=0-0.2 \mathrm{~s}, 0.2-0.6 \mathrm{~s}, 0.6-0.8 \mathrm{~s}, 0.8-1.0 \mathrm{~s}$, and $1.4-2.0 \mathrm{~s}$ with mean flow velocity $v=50 \mathrm{~m} / \mathrm{s}$. Figure 9(f) shows where the dominant frequency amplitude varies within different time intervals. Both the frequency of pressure fluctuation and the amplitude of each dominant frequency changed during the acoustic resonance excitation process. Before $0.8 \mathrm{~s}$, there were dominant frequency components of $50 \mathrm{~Hz}, 65 \mathrm{~Hz}$, and $185 \mathrm{~Hz}$ in the pressure fluctuations (Figures 9(a)-9(c)). Over time, the component frequency of $50 \mathrm{~Hz}$ was continuously amplified until it became the only dominant frequency (Figures $9(\mathrm{~d})$ and $9(\mathrm{e})$ ). The $185 \mathrm{~Hz}$ pressure fluctuation component increased slowly before $1.0 \mathrm{~s}$ slowly and was not obvious compared to the $50 \mathrm{~Hz}$ component. After $0.8 \mathrm{~s}$, the $65 \mathrm{~Hz}$ component disappeared in the dominant frequencies. The $185 \mathrm{~Hz}$ component did not disappear until $1.0 \mathrm{~s}$. It appears that the dominant acoustic resonance frequency is selectively 


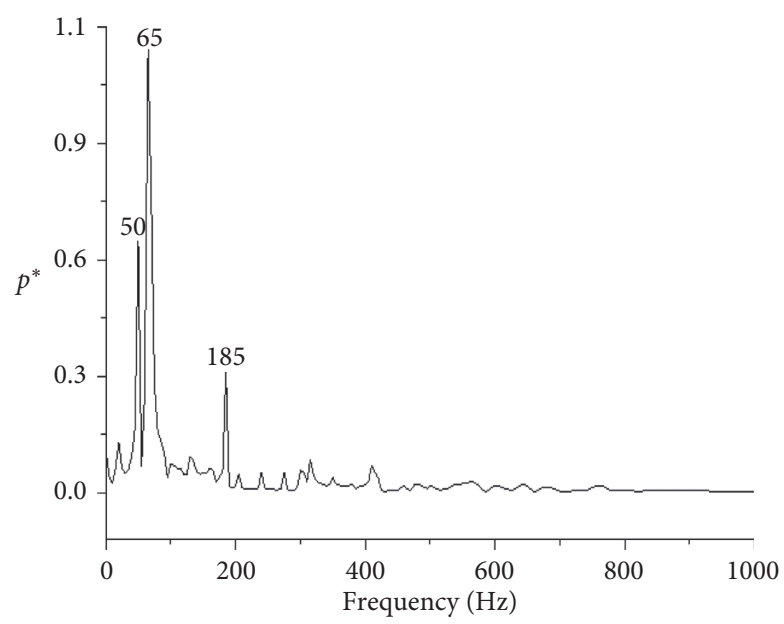

(a)

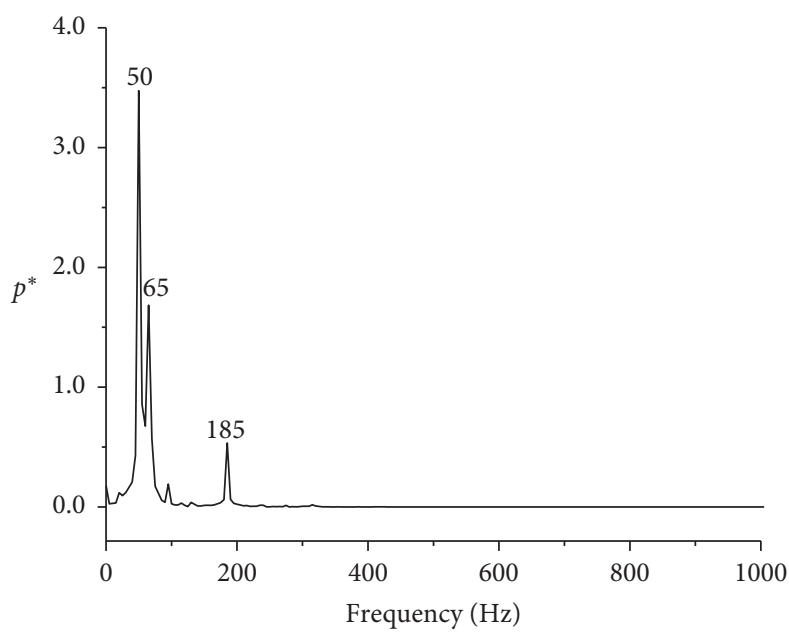

(c)

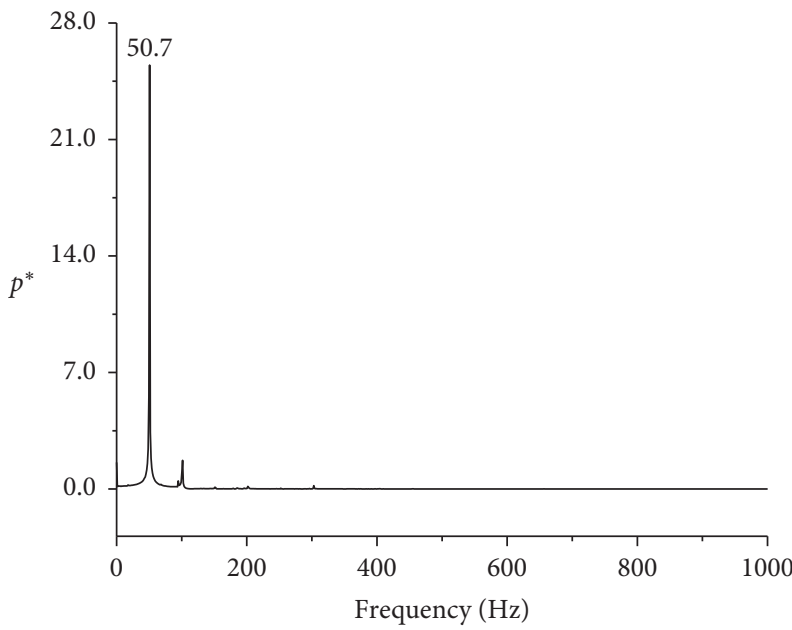

(e)

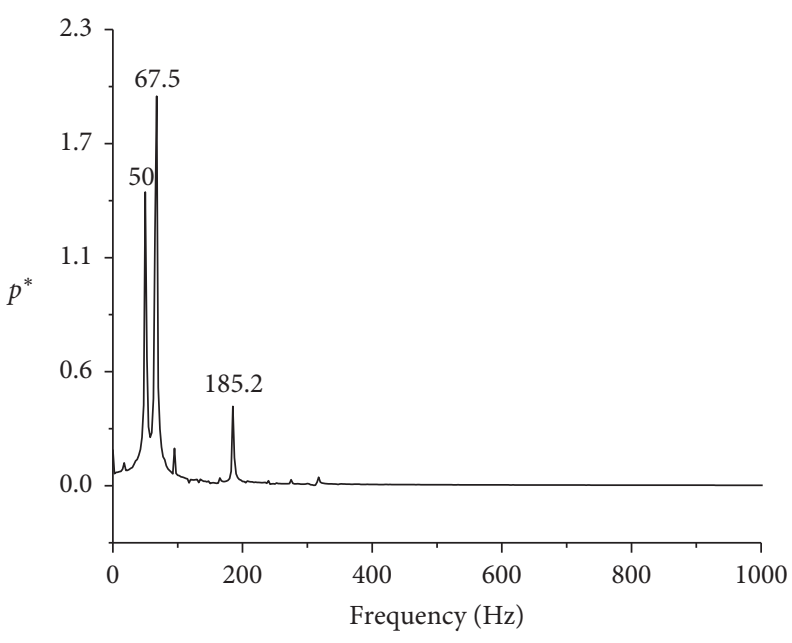

(b)

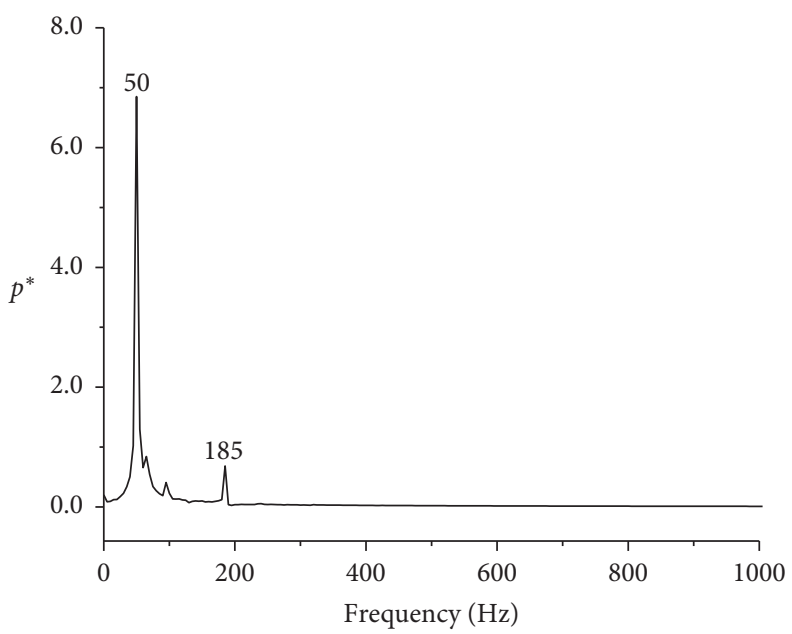

(d)

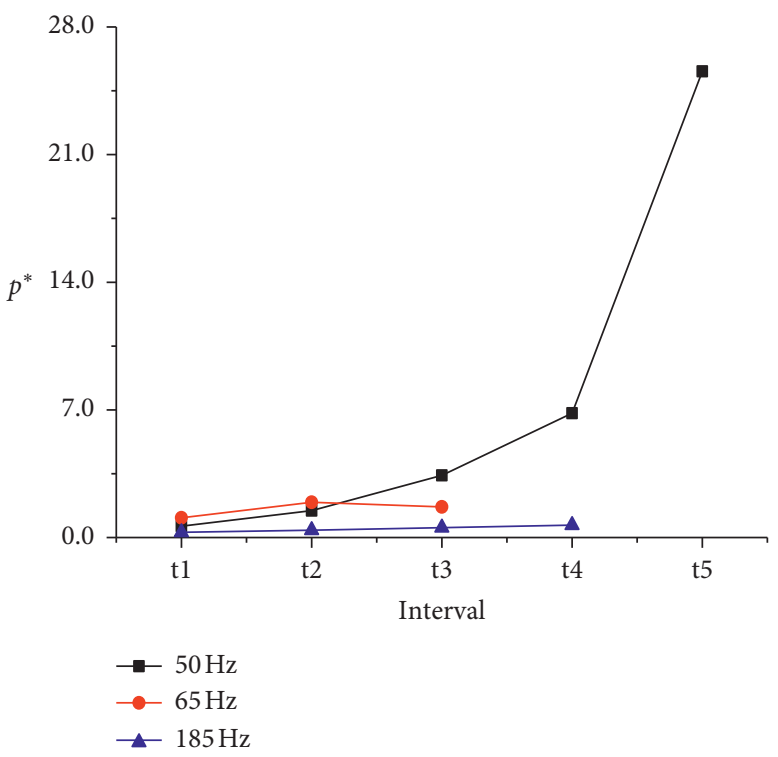

(f)

Figure 9: Pressure amplitude of dominant frequency with corresponding time intervals ( $v=50 \mathrm{~m} / \mathrm{s}$ ): (a) pressure fluctuation FFT analysis $(v=50 \mathrm{~m} / \mathrm{s}, t 1=0-0.2 \mathrm{~s})$, (b) pressure fluctuation FFT analysis $(v=50 \mathrm{~m} / \mathrm{s}, t 2=0.2-0.6 \mathrm{~s})$, (c) pressure fluctuation FFT analysis $(v=50 \mathrm{~m} / \mathrm{s}$, $t 3=0.6-0.8 \mathrm{~s})$, (d) pressure fluctuation FFT analysis $(v=50 \mathrm{~m} / \mathrm{s}, t 4=0.8-1.0 \mathrm{~s})$, (e) pressure fluctuation FFT analysis $(v=50 \mathrm{~m} / \mathrm{s}$, $t 5=1.4-2.0 \mathrm{~s})$, and (f) variation of pressure amplitude of dominant frequency with the corresponding time interval $(v=50 \mathrm{~m} / \mathrm{s})$. 
amplified in the excitation process while other components gradually disappear in the dominant frequencies.

The pressure fluctuations in other cases were analyzed using the same method to find that the excitation processes follow the same mode as the case of $v=50 \mathrm{~m} / \mathrm{s}$. As shown in Figures 5(a) and 5(b)), in the case of the mean flow velocity $v=10 \mathrm{~m} / \mathrm{s}$ and $15 \mathrm{~m} / \mathrm{s}$, the pressure fluctuation still had a component of approximately $50 \mathrm{~Hz}$ but no acoustic resonance occurred. Comparison with Figure 9(a) indicates that in the case of mean flow velocity $v=10 \mathrm{~m} / \mathrm{s}$ and $15 \mathrm{~m} / \mathrm{s}$, the power of the $50 \mathrm{~Hz}$ component was too small to excite the gas acoustic resonance in the closed side branch.

5.3. Mass Flow Rate into Branch. Figures 10(a)-10(c)) show the mass flow rate from the side branch mouth into the branch and the pressure of the monitoring point $p 1$ at three time intervals, $t 1-t 3$, where $t 1=0.1-0.12 \mathrm{~s}, t 2=0.6123-0.6323 \mathrm{~s}$, and $t 3=1.7-1.72 \mathrm{~s}$ in the case of mean flow velocity $v=40 \mathrm{~m} / \mathrm{s}$. All the time intervals contain 200 time steps allowing for an approximately sinusoidal wave time of an acoustic resonance period. As shown in Figure 10, the mass flow rate into the closed side branch was sinusoidal; the amplitude increased gradually with time until a stable acoustic resonance formed. The pressure at the bottom of the branch appears to be positively correlated with the mass flow rate into the branch. The pressure increased when the mass flow rate was positive, and vice versa. Acoustic resonance excitation is essentially a process in which the gas is pressed into and out of the branch in accordance with the sinusoidal mode.

5.4. Vortex Modes. The 200 time steps shown in Figure 10 were evenly divided into eight parts corresponding to the $0 \mathrm{~T} / 8-8 \mathrm{~T} / 8$ of the period. The vorticity on the $z=0$ plane of the dividing time points $(0 \mathrm{~T} / 8-8 \mathrm{~T} / 8)$ is shown in Figures 11-13 for the three time intervals and the velocity curl:

$$
\omega=\frac{\partial v}{\partial x}-\frac{\partial u}{\partial y} .
$$

The vorticity around the mouth of the branch increased with the flow time (Figures 11-13). The vorticity shows three distinct vortex modes at the three time intervals. At $t 1=0.1-0.12 \mathrm{~s}$, the detached shear layer beat the air at the branch mouth with low amplitudes; the vortex mode at most moments was similar to that of backward-facing step flow. At $t 2=0.6123-0.6323 \mathrm{~s}$, the detached shear layer formed a large vortex at the branch mouth which then impinged on the downstream wall. Small vortices were transmitted to the bottom of the branch at this time in a mode similar to shallow cavity flow. At $t 3=1.7-1.72 \mathrm{~s}$, the detached shear layer formed a larger vortex that was pushed out of the branch mouth without impingement on the downstream wall. This is a unique vortex mode.

We conclude that the acoustic resonance excitation process in the closed side branch can be divided into three steps: (1) the shear layer is free to detach from the upstream wall, (2) the detached shear layer interacts with the downstream wall at the branch mouth, and (3) the detached shear layers are coupled to the gas column resonance to form a periodic large vortex, which is excited in the branch as the shear layer no longer interacts with the downstream wall of the branch.

At the time $(6 \mathrm{~T} / 8 \longrightarrow 7 \mathrm{~T} / 8 \longrightarrow 8 \mathrm{~T} / 8 \longrightarrow 0 \mathrm{~T} / 8 \longrightarrow 1 \mathrm{~T} / 8)$, the shear layer gradually detached from the upstream wall to form a large vortex. This large vortex propagated from the main pipe to the branch, pressing the gas into the branch. We define this as the "former vortex." At the time $(2 \mathrm{~T} / 8 \longrightarrow 3 \mathrm{~T} /$ $8 \longrightarrow 4 \mathrm{~T} / 8 \longrightarrow 5 \mathrm{~T} / 8 \longrightarrow 6 \mathrm{~T} / 8$ ), the large vortex was gradually pushed to the main pipe by the compressed gas in the branch, accompanied by the gas moving out of the branch. The "later detached shear layer" from the upstream wall was brought upwards by the former vortex.

At 4T/8 (Figure 13(e)), the later detached shear layer was almost perpendicular to the flow direction. At 5T/8 (Figure 13(f)), the later detached shear layer was broken into an independent vortex (the later detached vortex) by the former detached vortex. At $6 \mathrm{~T} / 8$ (Figure 13(g)), the vorticity of the later detached vortex began to exceed the former detached vortex. At 7T/8 (Figure 13(h)), the trajectory of the later vortex and former vortex intersected; the two vortices integrated to a certain extent. In this unique mode, the vortex mode repeats periodically so that a stable acoustic resonance forms. We found that after this formation, the center of the large vortex is located in the upstream half around the branch mouth and the large eddy does not impinge the downstream wall.

5.5. Standing Wave. The pressure appeared to form a standing wave in the branch (Figure 14), so we further examined this aspect of the excitation process. The pressure along the branch axis at three time intervals is shown in Figure 15. At $t 1$, there is no obvious standing wave phenomenon but the wave node is clearly located near the inner side of the branch mouth. At $t 2$, the wave node obviously falls near the inner side of the branch mouth and the antinode is at the bottom of the branch. At $t 3$, the antinode is located at the bottom of the branch and there are no obvious nodes; all are located near to the outer side of the branch mouth. As shown in Figure 15(c), the pressure near the branch mouth steeply dropped off at this point.

The pressure drop was located in the branch and during the negative pressure time $(3 \mathrm{~T} / 8 \longrightarrow 7 \mathrm{~T} / 8)$ and in the main pipe (out of the branch) during the positive pressure time $(8 \mathrm{~T} / 8 \longrightarrow 2 \mathrm{~T} / 8)$. The reason for the irregularity of the standing wave node is observable in Figure 13. During the positive pressure time, the vortex moved toward the branch pipe, which contributed to the pressure drop; during the negative pressure time, the vortex moved toward the main pipe. The motion of the vortex prevented the sound source from being fixed. The distance from the branch mouth to the main pipe was greater than that to the branch, which is why equation (7) needs to contain the correction coefficient $L e$.

The frequency validation result $f_{1 c}=50.8 \mathrm{~Hz}$, which is smaller than the result predicted by equation (7), $f_{1}=61.8 \mathrm{~Hz}$. This is because the acoustic vortex (sound 


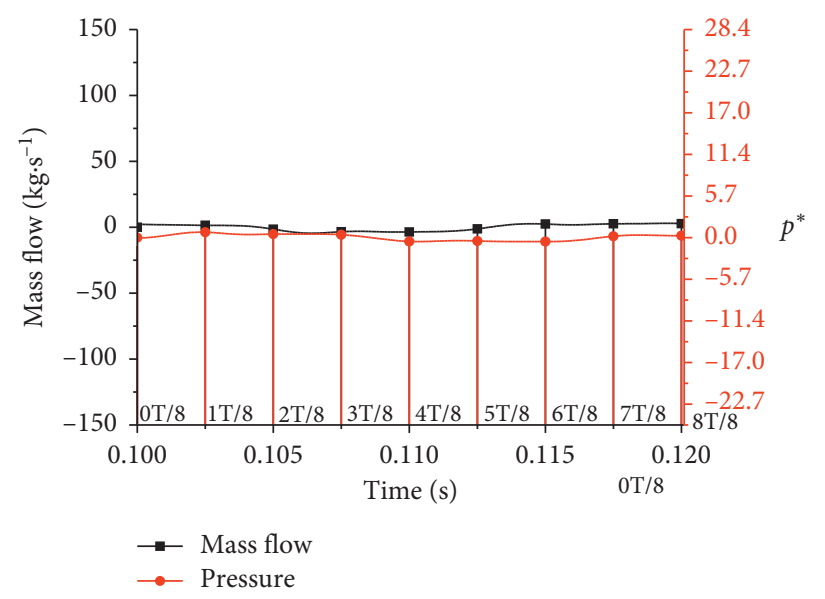

(a)

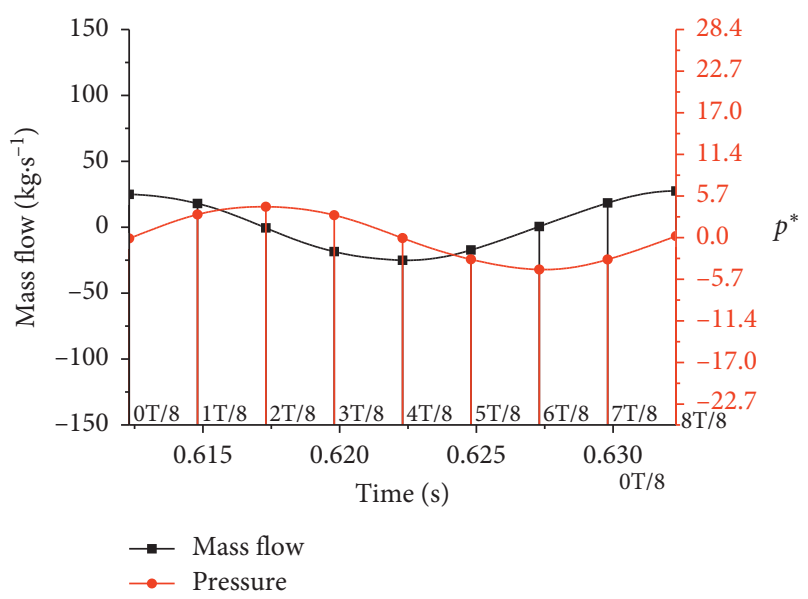

(b)

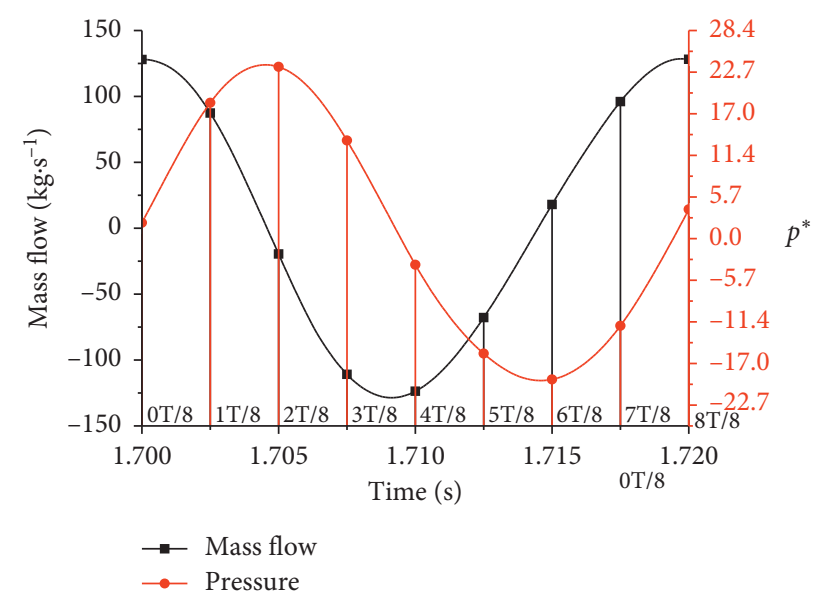

(c)

FiguRE 10: Mass flow rate into branch and pressure of monitoring point $p 1(v=40 \mathrm{~m} / \mathrm{s}):$ (a) $t 1=0.1 \mathrm{~s}-0.12 \mathrm{~s},(\mathrm{~b}) t 2=0.6123 \mathrm{~s}-0.6323 \mathrm{~s}$, and (c) $t 3=1.7 \mathrm{~s}-1.72 \mathrm{~s}$

source) moved toward the main pipe, so that the wavelength of the standing wave increased and the frequency decreased significantly. At the time intervals of $t 1$ and $t 2$, the standing wave length was shorter due to the effect of the $65 \mathrm{~Hz}$ pressure fluctuation component. Figures 9(a) and 9 (c) illustrate this phenomenon. At the t1 time interval, $65 \mathrm{~Hz}$ was the dominant frequency. At t2, the $65 \mathrm{~Hz}$ component persisted but had become the secondary frequency while the approximately $50 \mathrm{~Hz}$ component became the dominant frequency. At $\mathrm{t} 3$, the $65 \mathrm{~Hz}$ component disappeared and the $50 \mathrm{~Hz}$ component was completely dominant.

5.6. Phase Plane Characteristics. Shaaban and Mohany [40] pointed out that the energy transfer from the flow field to the sound field can overcome the energy taken away by sound damping and radiation, maintaining high-pressure fluctuations. The mechanism of self-excited resonance in the closed side branch is similar to that around inline cylinders.

Figure 16 shows the phase trajectories of all the cases in the phase plane, where it appears that whether acoustic resonance is formed determines the final shape of the phase trajectory. When acoustic resonance can be formed, the limit cycle exists and the phase trajectory eventually converges to the limit cycle. When acoustic resonance cannot form, the limit cycle does not exist and the phase trajectory converges to the equilibrium point. In the case of the mean flow velocity $v=10 \mathrm{~m} / \mathrm{s}$ and $20 \mathrm{~m} / \mathrm{s}$, the phase trajectory tended toward the coordinate origin $(0,0)$ from the outside to the center, indicating that the equilibrium point fell at the approximate coordinate origin (Figures 16(a) and 16(b)). In the other cases, the phase trajectories converged to a limit cycle from the center to the outside. A curve direction toward the center (limit cycle) reflects the consumption (production) of source energy. Again, whether acoustic resonance occurs depends on whether the sound source energy is greater than the energy taken away by the sound damping and radiation, and the area of this curve illustrates the strength of the source.

However, in the cases of convergence toward the limit cycle (Figure 16(c)-16(h)), different oval shapes appear to form. In cases of mean flow velocity $v=30 \mathrm{~m} / \mathrm{s}, 40 \mathrm{~m} / \mathrm{s}$, or $50 \mathrm{~m} / \mathrm{s}$, the oval long axis is on the left side; with mean flow 


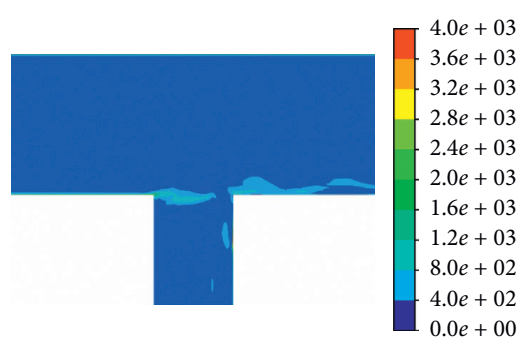

(a)

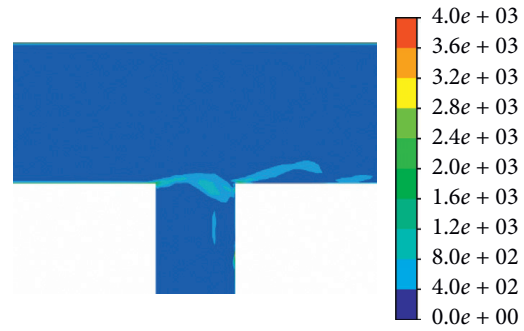

(d)

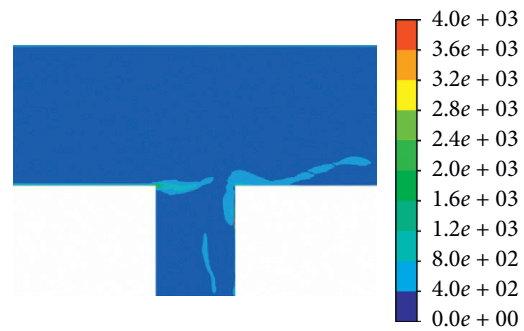

(g)

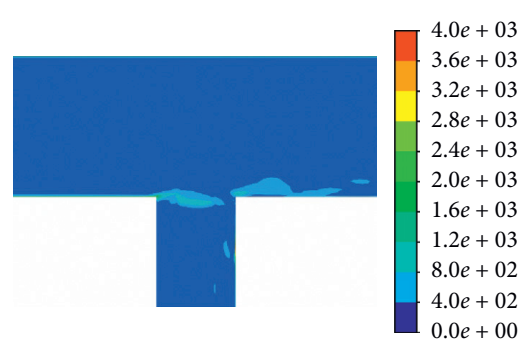

(b)

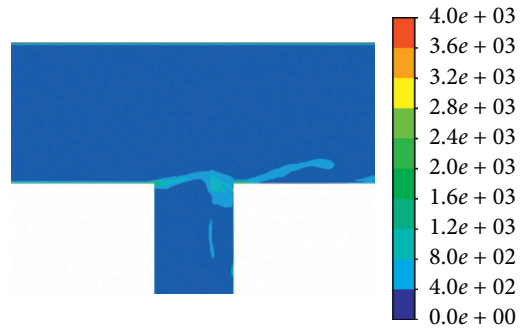

(e)

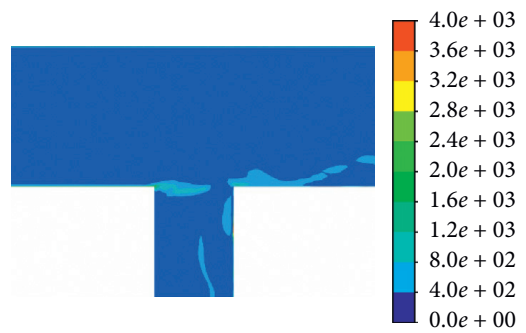

(h)

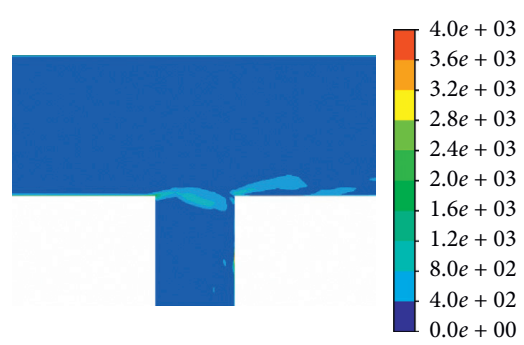

(c)

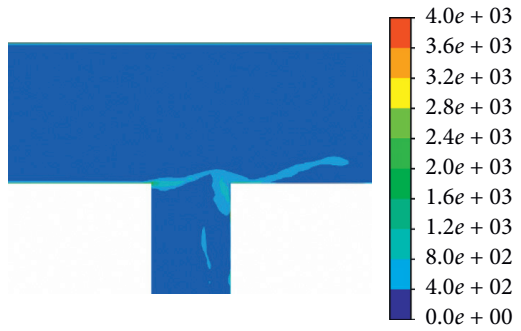

(f)

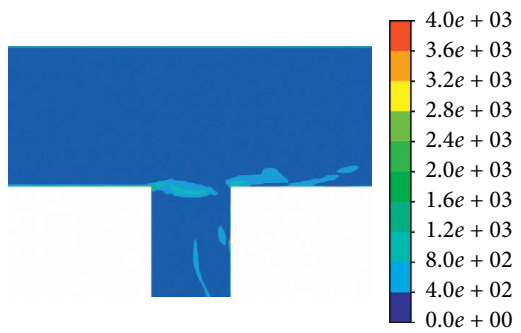

(i)

Figure 11: Vorticity $\left(t 1=0.1-0.12 \mathrm{~s}\right.$ unit: $\mathrm{s}^{-1}$ ): (a) 0T/8, (b) 1T/8, (c) 2T/8, (d) 3T/8, (e) 4T/8, (f) 5T/8, (g) 6T/8, (h) 7T/8 and (i) $8 \mathrm{~T} / 8$.

velocity $v=60 \mathrm{~m} / \mathrm{s}$ or $70 \mathrm{~m} / \mathrm{s}$, the oval long axis is on the right side. The limit cycle is a regular oval in the case of the mean flow velocity $v=20 \mathrm{~m} / \mathrm{s}$ and $30 \mathrm{~m} / \mathrm{s}$. The limit cycle is relatively regular but oval in the case of the mean flow velocity $v=40 \mathrm{~m} / \mathrm{s}$ and an irregular oval in the case of the mean flow velocity $v=50 \mathrm{~m} / \mathrm{s}$ and $60 \mathrm{~m} / \mathrm{s}$. The limit cycle is also relatively oval in the case of the mean flow velocity $v=70 \mathrm{~m} / \mathrm{s}$. This phase shape ovality was expected due to harmonics, as is also shown in Figure 7.

Figure 16 with Figure 7 together show that the shape of the limit cycle is affected by the second-order dominant frequency component. The limit cycle appears to grow more irregular the more obvious the second-order dominant frequency component is. Furthermore, the influence of the second-order dominant frequency intensifies when closer to the mean flow velocity; the limit cycle also grows more irregular. The phase trajectories were similar to the shape of the final limit cycle in all cases except for mean flow velocity $v=60 \mathrm{~m} / \mathrm{s}$ (Figure $16(\mathrm{~g})$ ). In the case of the mean flow velocity $v=60 \mathrm{~m} / \mathrm{s}$ (Figure $16(\mathrm{~g})$ ), the phase trajectory in the phase plane first shows an oval long axis along the direction of $Y$, and then ultimately the oval long axis falls along the direction of $X$. These two different trends illustrate that the excitation process of the oval long axis is relatively unstable. The amount of regularity should indicate whether the coupling is stable or not.
The stability of the above process is related to the approximation of the second-order acoustic resonance velocity. The phase trajectory appears to grow less stable as the mean flow velocity grows closer to the second-order acoustic resonance velocity. As shown in Figure 16(g), the phase trajectory was extremely unstable in the case of the mean flow $v=60 \mathrm{~m} / \mathrm{s}$. This indicates that the instability factor increased with flow velocity during the transition from firstorder acoustic resonance to second-order acoustic resonance. However, the process recovered a great deal of its stability in the case of mean flow $v=70 \mathrm{~m} / \mathrm{s}$.

\subsection{Self-Excited Vibration Characteristics of Acoustic} Resonance. The acoustic resonance in the closed side branch presented self-excited vibration (SEV) in our simulation. The SEV and acoustic resonance characteristics discussed above suggest that acoustic resonance is an SEV system in which the pressure fluctuation of the gas is a vibration variable. In an actual system, the gas conveyed in the pipeline and the pipe can also be regarded as an SEV system. We did not build vibration of the pipeline into our simulation, so this is not discussed here.

The mathematical model of the SEV system must be a nonlinear differential equation [29]. As an SEV system, the acoustic resonance in the closed side branch shows pure- 


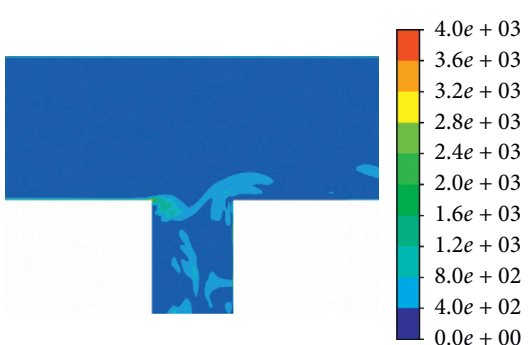

(a)

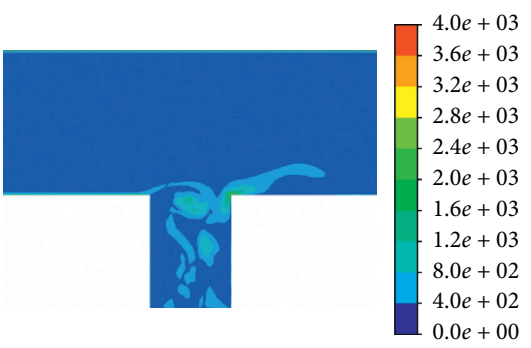

(d)

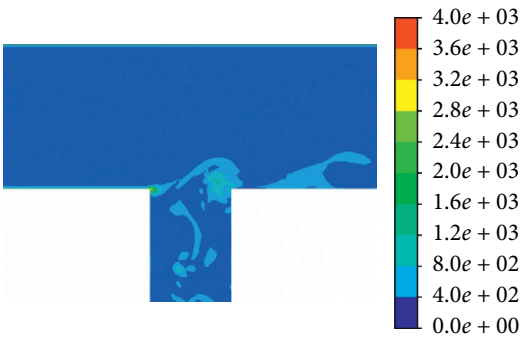

(g)

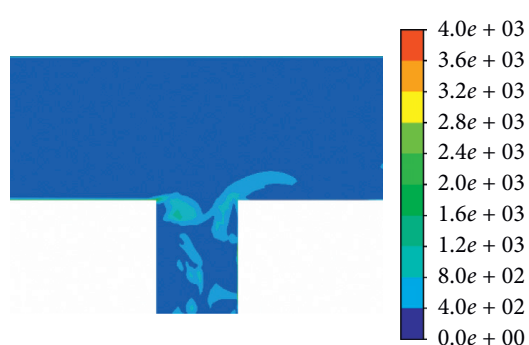

(b)

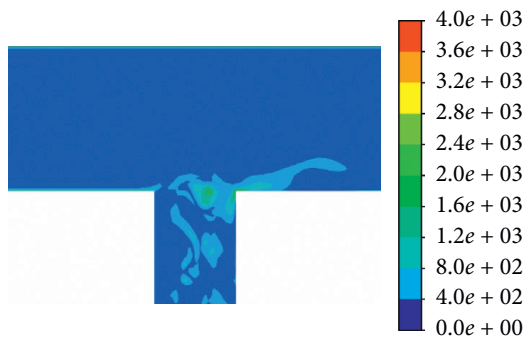

(e)

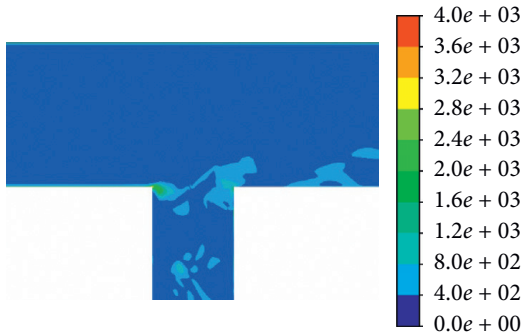

(h)

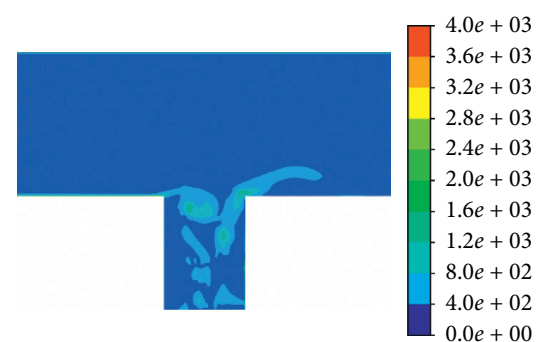

(c)

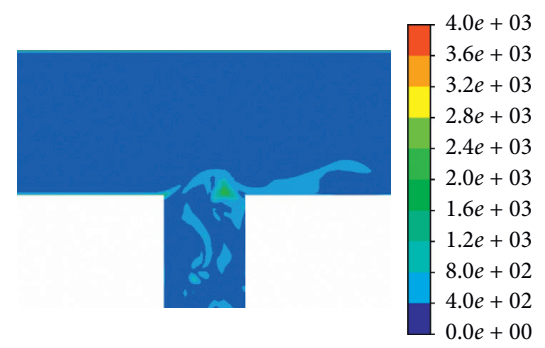

(f)

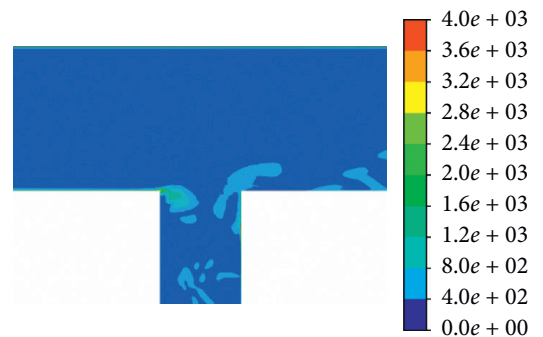

(i)

Figure 12: Vorticity $\left(t 2=0.6123-0.6323 \mathrm{~s} \mathrm{unit:}^{-1}\right)$ : (a) 0T/8, (b) 1T/8, (c) 2T/8, (d) 3T/8, (e) 4T/8, (f) 5T/8, (g) 6T/8, (h) 7T/8, and (i) 8T/8.

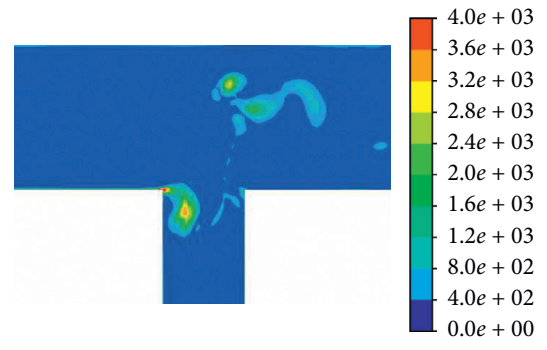

(a)

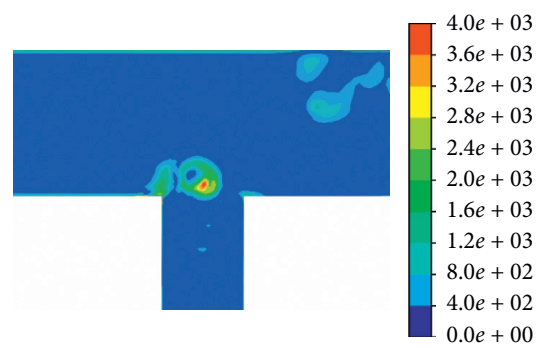

(d)

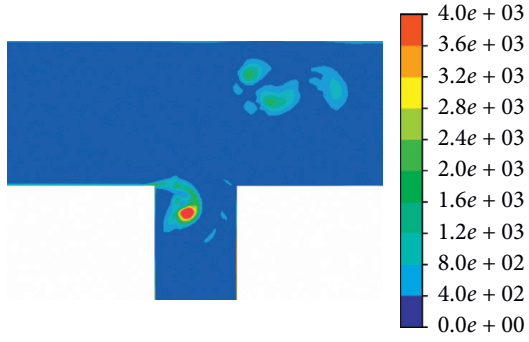

(b)

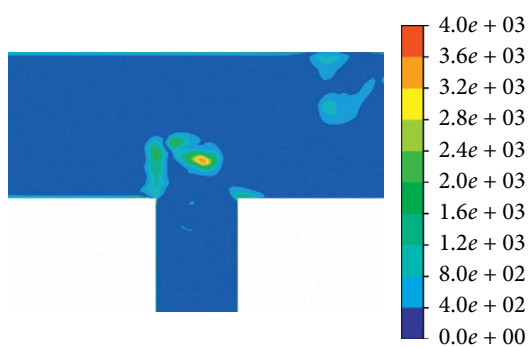

(e)

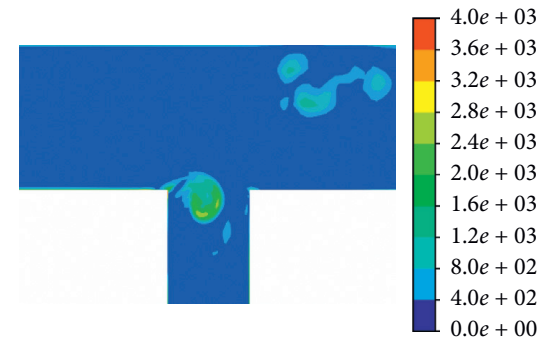

(c)

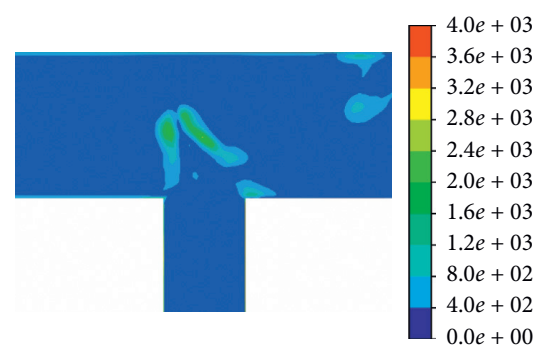

(f)

FIgURE 13: Continued. 


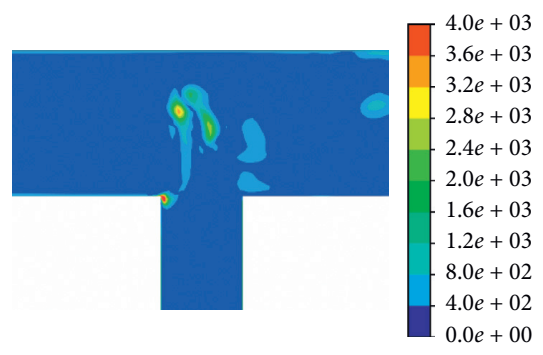

(g)

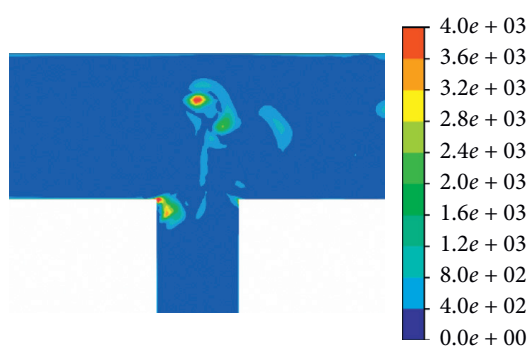

(h)

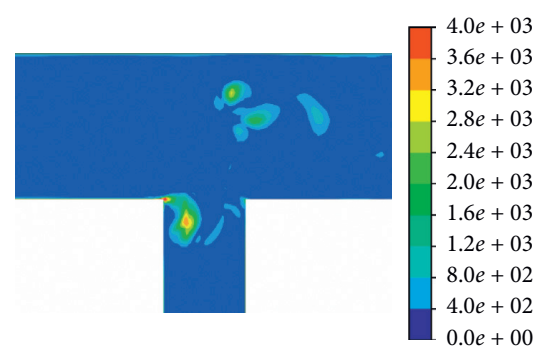

(i)

Figure 13: Vorticity $\left(t 3=1.7-1.72 \mathrm{~s} u n i t: ~^{-1}\right.$ ): (a) 0T/8, (b) 1T/8, (c) 2T/8, (d) 3T/8, (e) 4T/8, (f) 5T/8, (g) 6T/8, (h) 7T/8, and (i) $8 \mathrm{~T} / 8$.

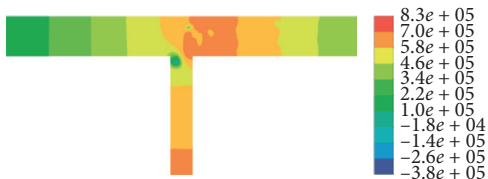

(a)

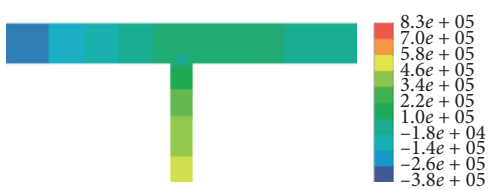

(d)

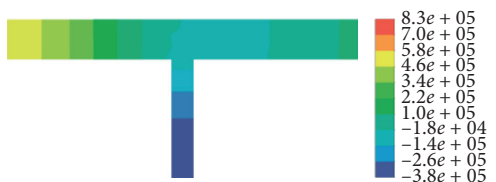

(g)

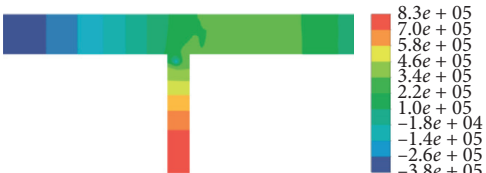

(b)

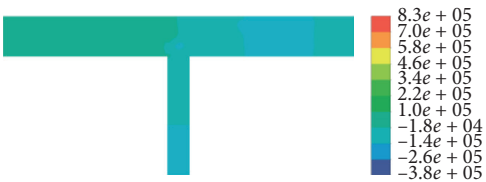

(e)

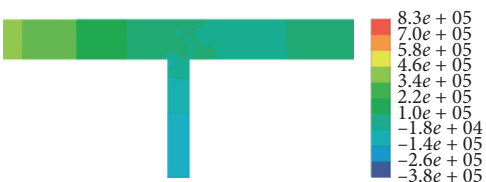

(h)

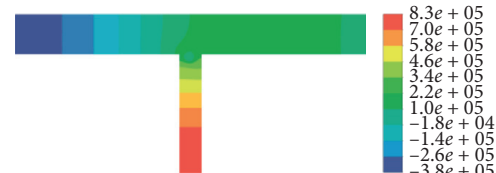

(c)

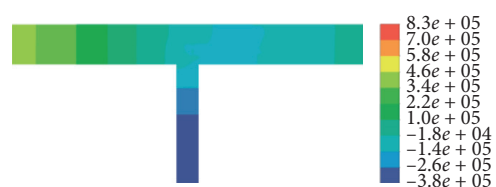

(f)

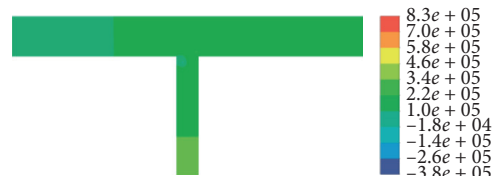

(i)

Figure 14: Pressure contour ( $t 3=1.7$ s-1.72 s, unit: Pa): (a) 0T/8, (b) $1 \mathrm{~T} / 8$, (c) $2 \mathrm{~T} / 8$, (d) $3 \mathrm{~T} / 8$, (e) 4 T/8, (f) $5 \mathrm{~T} / 8$, (g) $6 \mathrm{~T} / 8$, (h) $7 \mathrm{~T} / 8$, and (i) $8 \mathrm{~T} / 8$.

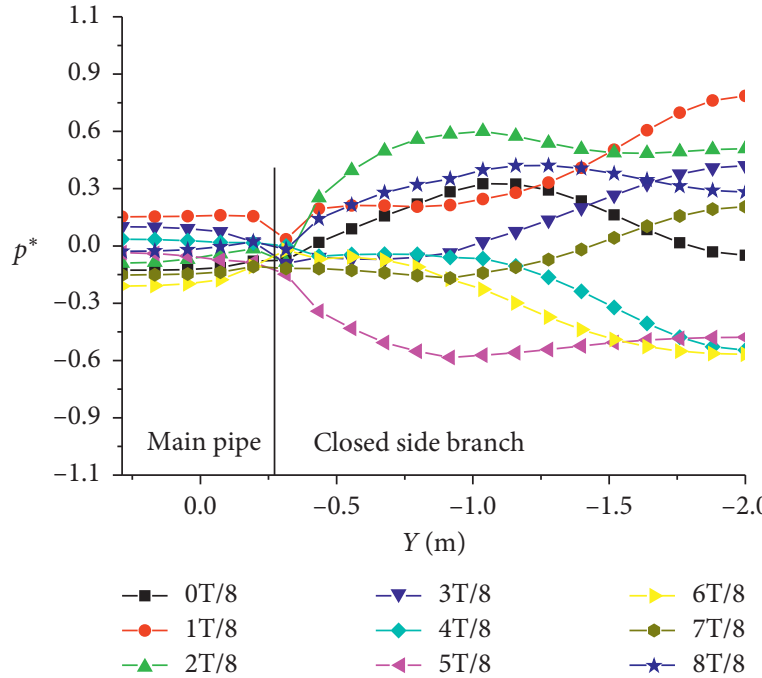

(a)

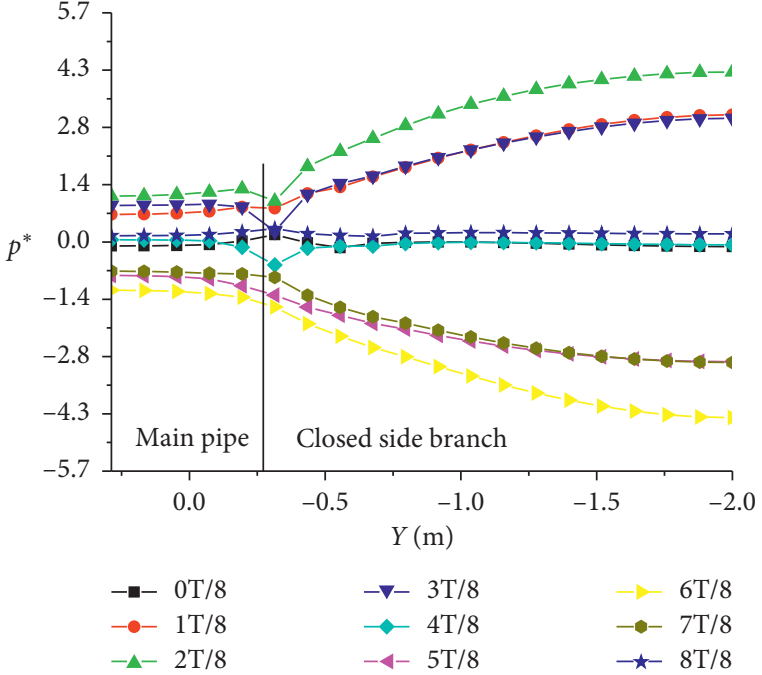

(b)

Figure 15: Continued. 


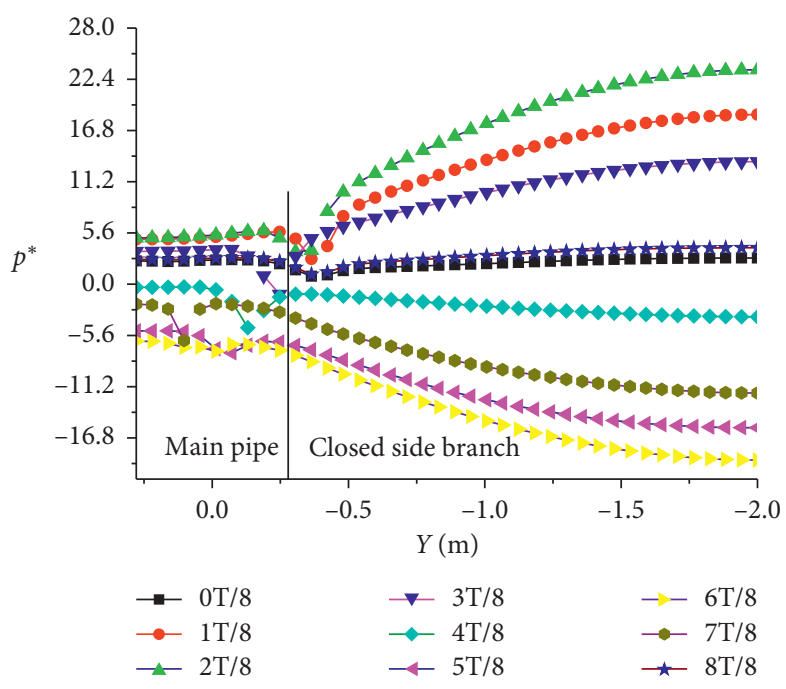

(c)

FIGURE 15: Standing wave along closed side branch: (a) $t 1=0.1 \mathrm{~s}-0.12 \mathrm{~s}$, (b) $t 2=0.6123 \mathrm{~s}-0.6323 \mathrm{~s}$, and (c) $t 3=1.7 \mathrm{~s}-1.72 \mathrm{~s}$.

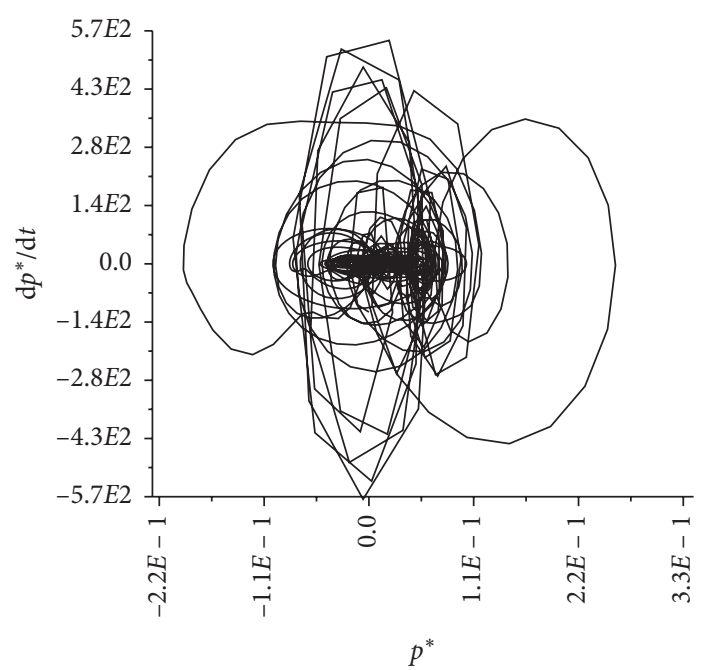

(a)

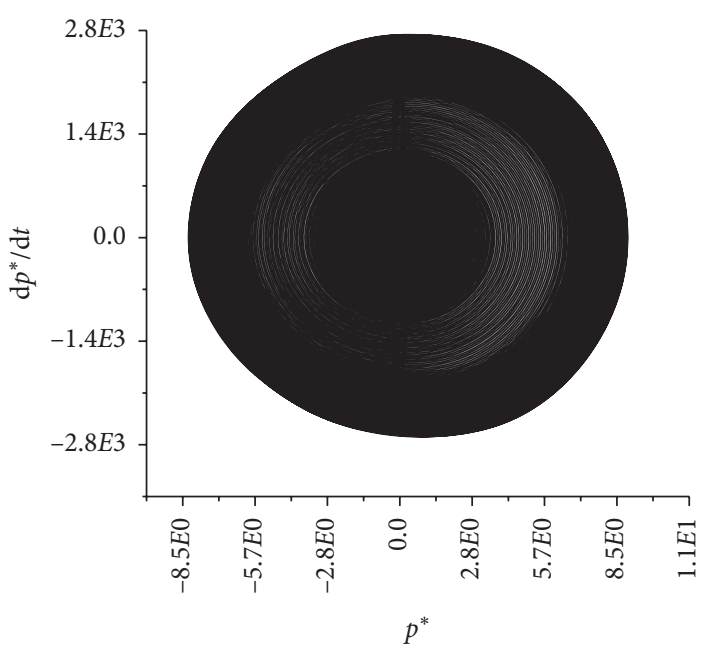

(c)

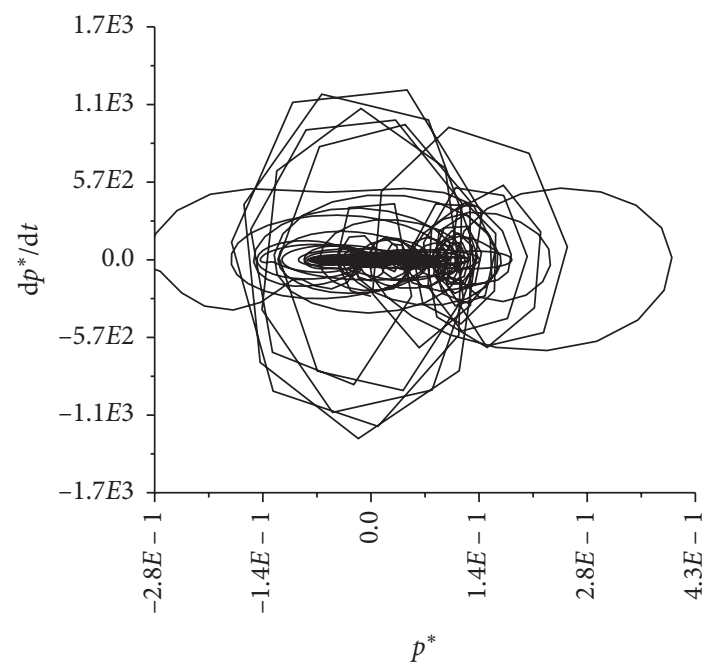

(b)

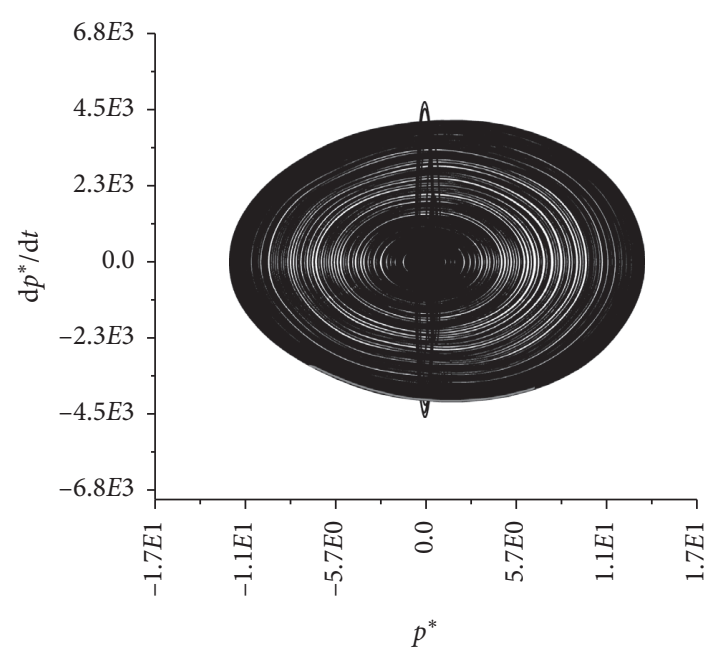

(d)

FIgUre 16: Continued. 


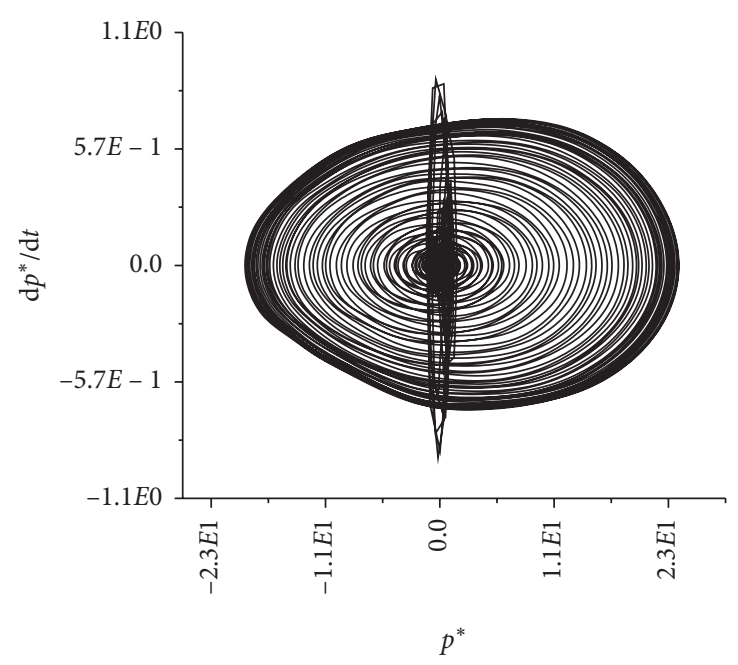

(e)

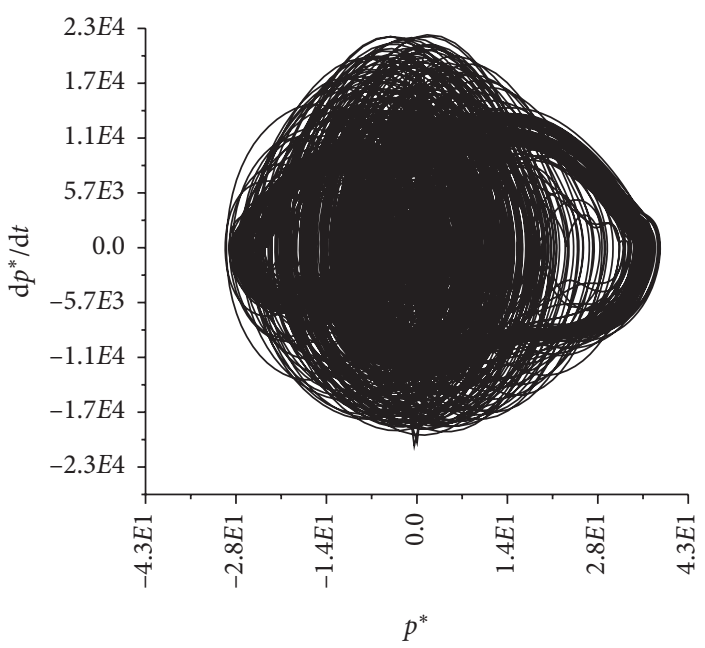

(g)

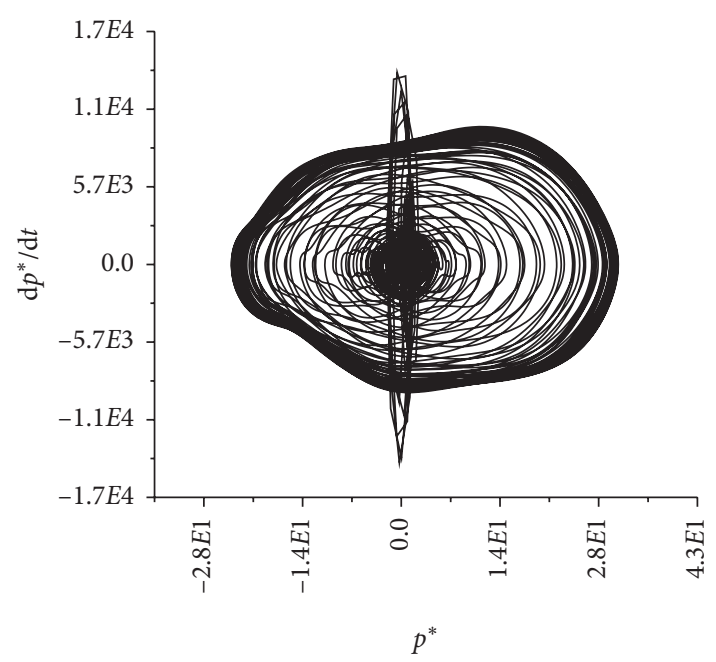

(f)

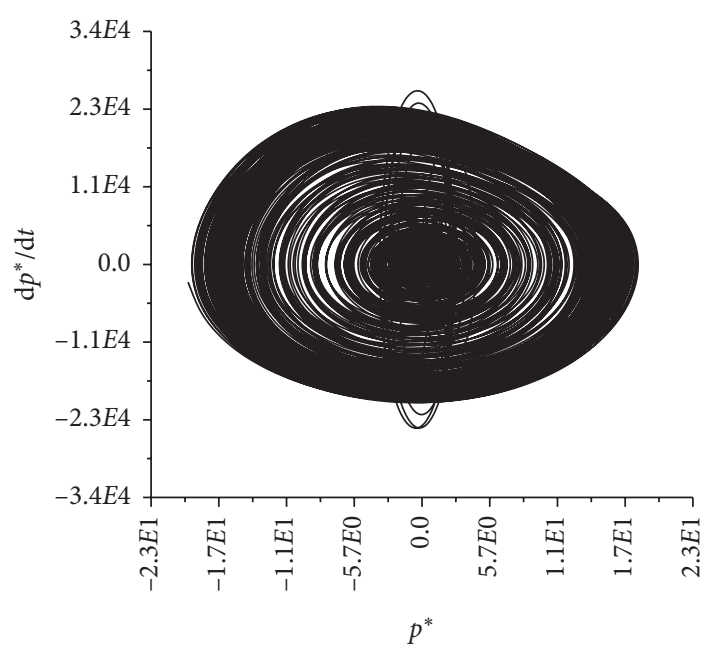

(h)

Figure 16: Phase plane: (a) $v=10 \mathrm{~m} / \mathrm{s}$, (b) $v=15 \mathrm{~m} / \mathrm{s}$, (c) $v=20 \mathrm{~m} / \mathrm{s}$, (d) $v=30 \mathrm{~m} / \mathrm{s}$, (e) $v=40 \mathrm{~m} / \mathrm{s}$, (f) $v=50 \mathrm{~m} / \mathrm{s}$, (g) $v=60 \mathrm{~m} / \mathrm{s}$, and (h) $v=70 \mathrm{~m} / \mathrm{s}$.

tone characteristics which approximate the simple harmonic function, so it is a quasiharmonic SEV system (weakly nonlinear autonomous system). The natural frequency of a weakly nonlinear SEV system is $\omega_{0}$, so the equation of motion is as follows:

$$
\ddot{x}+\omega_{0}^{2} x=\mu f(x, \dot{x}),
$$

where $f(x, \dot{x})$ is a nonlinear function that does not contain the linear term of $x$, and $\mu$ is a small constant. A larger $\mu$ value makes the phase trajectory tend toward the limit cycle (or the equilibrium point) more quickly. Our simulation results show that the mean flow velocity in the main pipe $v$ affects the speed of the phase trajectory approaching the limit cycle as well as the shape of the limit cycle. We conclude that the mean flow velocity $v$ in the main pipe should be within the nonlinear damping force system of SEV system equation (12) and positively correlated with $\mu$. The length of the closed side branch and the acoustic velocity of the fluid determine the frequency of acoustic resonance (equation (7)), so the length of the branch and the acoustic velocity determine the $\omega_{0}$ in equation (12).

\section{Conclusion}

We simulated acoustic resonance in a single closed side branch of a natural gas pipeline based on a realizable $k-\varepsilon$ DDES turbulence model in this study. We investigated flow characteristics of the acoustic resonance excitation process accordingly via the phase plane method from the SEV perspective. Our conclusions can be summarized as follows:

(1) In the excitation process, the dominant acoustic resonance frequency of the closed side branch can be selected and amplified while other components gradually disappear. The acoustic resonance frequency ultimately dominates the pure-tone characteristic. 
(2) A large vortex forms around the branch mouth which brings the gas in to (out of) the branch pipe with a sinusoidal periodic mass. The vortex mode is unique from the background step flow and shallow cavity flow, but the excitation process successively experiences the characteristics of both flows.

(3) The excitation time and stability of acoustic resonance are related to the mean flow velocity. The excitation time is negatively correlated with the mean flow velocity; it is shorter when the mean flow velocity is relatively large. The stability of acoustic resonance decreases with the mean flow velocity during the transition from the first-order acoustic resonance to the second order, and then the secondorder acoustic resonance recovers the stability.

(4) Acoustic resonance is an SEV system. We recommend modeling the acoustic resonance as a weakly nonlinear SEV equation.

\section{Data Availability}

The data used to support the findings of this study are available from the corresponding author upon request.

\section{Conflicts of Interest}

The authors declare that they have no conflicts of interest.

\section{Acknowledgments}

This research acknowledges the financial support provided by the China National Key Research and Development Plan (no. 2016YFC0802105).

\section{References}

[1] M. Su, Z. Zhang, Y. Zhu, and D. Zha, "Data-driven natural gas spot price forecasting with least squares regression boosting algorithm," Energies, vol. 12, no. 6, p. 1094, 2019.

[2] L. S. Tiewsoh, J. Jirásek, and M. Sivek, "Electricity generation in India: Present state, future outlook and policy implications," Energies, vol. 12, no. 7, p. 1361, 2019.

[3] R. M. Baldwin and H. R. Simmons, "Flow-induced vibration in safety relief valves," Journal of Pressure Vessel Technology, vol. 108, no. 3, pp. 267-272, 2009.

[4] G. DeBoo, R. Gesior, K. Ramsden, and B. Strub, "Identification of quad cities main steam line acoustic sources and vibration reduction," American Society of Mechanical Engineers, vol. 4, pp. 485-491, 2008.

[5] P. C. Kriesels, M. C. A. M. Peters, A. Hirschberg et al., "High amplitude vortex-induced pulsations in a gas transport system," Journal of Sound and Vibration, vol. 184, no. 2, pp. 343-368, 1995.

[6] D. Tonon, A. Hirschberg, J. Golliard, and S. Ziada, "Aeroacoustics of pipe systems with closed branches," Noise Notes, vol. 10, no. 3, pp. 27-88, 2011.

[7] C. Q. Howard and R. A. Craig, "Noise reduction using a quarter wave tube with different orifice geometries," Applied Acoustics, vol. 76, pp. 180-186, 2014.

[8] Y. Xiao, W. Zhao, H. Gu, and X. Gao, "Effects of branch length and chamfer on flow-induced acoustic resonance in closed side branches," Annals of Nuclear Energy, vol. 121, pp. 186193, 2018.

[9] M. Shaaban and A. Mohany, "Passive control of flow-excited acoustic resonance in rectangular cavities using upstream mounted blocks," Experiments in Fluids, vol. 56, no. 4, 2015.

[10] B. D. Knotts and A. Selamet, "Suppression of flow-acoustic coupling in sidebranch ducts by interface modification," Journal of Sound and Vibration, vol. 265, no. 5, pp. 1025-1045, 2003.

[11] S. Ziada and S. Shine, "Strouhal numbers of flow-excited acoustic resonance of closed side branches," Journal of Fluids and Structures, vol. 13, no. 1, pp. 127-142, 1999.

[12] K. Okuyama, A. Tamura, S. Takahashi, M. Ohtsuka, and M. Tsubaki, "Flow-induced acoustic resonance at the mouth of one or two side branches," Nuclear Engineering and Design, vol. 249, pp. 154-158, 2012.

[13] S. Ziada, A. Scott, and D. Arthurs, "Acoustic excitation by flow in T-junctions," Journal of Pressure Vessel Technology, vol. 129, no. 1, pp. 14-20, 2007.

[14] A. Powell, "Theory of vortex sound," The Journal of the Acoustical Society of America, vol. 36, no. 1, pp. 177-195, 1964.

[15] M. S. Howe, "Vorticity and the theory of aerodynamic sound," Journal of Engineering Mathematics, vol. 27, no. 3, pp. 143204, 2001.

[16] A. Michalke, "On spatially growing disturbances in an inviscid shear layer," Journal of Fluid Mechanics, vol. 23, no. 3, p. 521, 1965.

[17] M. Karlsson and M. Åbom, "Aeroacoustics of $T$-junctions - an experimental investigation," Journal of Sound and Vibration, vol. 329, no. 10, pp. 1793-1808, 2010.

[18] H. R. Graf and S. Ziada, "Excitation source of a side-branch shear layer," Journal of Sound and Vibration, vol. 329, no. 10, pp. 1793-1808, 2010.

[19] S. Ziada, "A flow visualization study of flow- acoustic coupling at the mouth of a resonant side-branch," Journal of Fluids and Structures, vol. 8, no. 4, pp. 391-416, 1994.

[20] Y. R. Li, S. Someya, and K. Okamoto, "An experimental investigation of flow-induced acoustic resonance and flow field in a closed side branch system using a high time-resolved PIV technique," Journal of Visualization, vol. 13, no. 1, pp. 7-16, 2010.

[21] Y. Li, S. Someya, K. Okamoto, T. Inagaki, and Y. Nishi, "Study on flow-induced acoustic resonance in symmetrically located side-branches using dynamic PIV technique," Journal of Fluid Science and Technology, vol. 9, no. 2, 2014.

[22] Y. Li, S. Someya, K. Okamoto, T. Inagaki, and Y. Nishi, "Visualization study of flow-excited acoustic resonance in closed tandem side branches using high time-resolved particle image velocimetry," Journal of Mechanical Science and Technology, vol. 29, no. 3, pp. 989-999, 2015.

[23] E. Salt, S. Mohamed, D. Arthurs, and S. Ziada, "Identification of aeroacoustic sources in a $T$-junction," American Society of Mechanical Engineers, vol. 4, 2014.

[24] E. Salt, S. Mohamed, D. Arthurs, and S. Ziada, "Aeroacoustic sources generated by flow-sound interaction in a $T$-junction," Journal of Fluids and Structures, vol. 51, pp. 116-131, 2014.

[25] Y. Xiao, H. Gu, X. Gao, H. Zhang, and W. Zhao, "Flow visualization study of flow-induced acoustic resonance in closed side branches," Annals of Nuclear Energy, vol. 121, pp. 186193, 2018.

[26] A. Tamura, K. Okuyama, S. Takahashi, and M. Ohtsuka, "Development of numerical analysis method of flow-acoustic resonance in stub pipes of safety relief valves," Journal of 
Nuclear Science and Technology, vol. 49, no. 8, pp. 793-803, 2012.

[27] P. M. Radavich, A. Selamet, and J. M. Novak, "A computational approach for flow-acoustic coupling in closed side branches," The Journal of the Acoustical Society of America, vol. 109, no. 4, pp. 1343-1353, 2002.

[28] S. Dequand, S. J. Hulshoff, and A. Hirschberg, "Self-sustained oscillations in a closed side branch system," Journal of Sound and Vibration, 2003;vol. 265, no. 2, pp. 359-386.

[29] W. Ding, Self-excited Vibration: Theory, Paradigms, and Research Methods, Springer Science and Business Media, Berlin, Germany, 2013.

[30] V. M. Calo, Y. Bazilevs, T. J. R. Hughes, and R. Moser, "Turbulence modeling for large eddy simulations," Computer Methods in Applied Mechanics and Engineering, vol. 199, no. 13-16, p. 779, 2010.

[31] M. Lesieur and O. Métais, "New trends in large-eddy simulations of turbulence," Annual Review of Fluid Mechanics, vol. 28, no. 1, pp. 45-82, 1996.

[32] P. R. Spalart, W. H. Jou, M. K. Strelets, and S. R. Allmaras, "Comments on the feasibility of LES for wings and on a hybrid RANS/LES approach," in Proceedings of the First AFOSR International Conference on DNS/LES, Ruston, LA, USA, August 1997.

[33] T. H. Shih, W. W. Liou, A. Shabbir, Z. Yang, and J. Zhu, "A new $\mathrm{k}-\varepsilon$ eddy viscosity model for high Reynolds number turbulent flows," Computers and Fluids, vol. 24, no. 3, pp. 227-238, 1995.

[34] P. R. Spalart, S. Deck, M. L. Shur, K. D. Squires, M. K. Strelets, and A. Travin, "A new version of detached-eddy simulation, resistant to ambiguous grid densities," Theoretical and Computational Fluid Dynamics, vol. 20, no. 3, pp. 181-195, 2006.

[35] X. M. Tan, P. P. Xie, Z. G. Yang, and J. Y. Gao, “Adaptability of turbulence models for pantograph aerodynamic noise simulation," Shock and Vibration, vol. 2019, pp. 1-20, Article ID 6405809, 2019.

[36] Y. Zhang, J. Zhang, T. Li, L. Zhang, and W. Zhang, "Research on aerodynamic noise reduction for high-speed trains," Shock and Vibration, vol. 2016, pp. 1-21, Article ID 6031893, 2016.

[37] S. Takahashi, M. Ohtsuka, K. Okuyama, T. Ito, and K. Yoshikawa, "Experimental study of acoustic and flowinduced vibrations in BWR main steam lines and steam dryers," American Society of Mechanical Engineers, vol. 4, pp. 41-47, 2008.

[38] Q. Yuan, B. Yu, J. Li, D. Han, and W. Zhang, "Study on the restart algorithm for a buried hot oil pipeline based on wavelet collocation method," International Journal of Heat and Mass Transfer, vol. 125, pp. 891-907, 2018.

[39] K. Zhang, J. Li, B. Yu, D. Han, and Y. Chen, "Fast prediction of the replacement process of oil vapor in horizontal tank and its improved safety evaluation method," Process Safety and Environmental Protection, vol. 122, pp. 298-306, 2019.

[40] M. Shaaban and A. Mohany, "Experimental study of the selfexcited resonance effect on the dynamic lift and flow structure around inline cylinders," Journal of Fluids and Structures, vol. 96, Article ID 103015, 2020. 